\title{
Multiplicities of simple closed geodesics and hypersurfaces in Teichmüller space
}

\author{
GREG MCSHANE \\ Hugo PARLIER
}

\begin{abstract}
Using geodesic length functions, we define a natural family of real codimension 1 subvarieties of Teichmüller space, namely the subsets where the lengths of two distinct simple closed geodesics are of equal length. We investigate the point set topology of the union of all such hypersurfaces using elementary methods. Finally, this analysis is applied to investigate the nature of the Markoff conjecture.
\end{abstract}

57M50; 58D99

\section{Introduction}

We define the simple length spectrum of a Riemann surface of genus $g$ with $n$ totally geodesic boundary components to be the set of lengths of simple closed geodesics counted with multiplicities. As the metric varies, the length spectrum changes. We are interested in three questions.

Is there a surface for which all the multiplicities are 1 ?

How big is the set of such surfaces?

Is it possible to deform a surface such that the multiplicity stays 1 for all simple geodesics?

The answer to the first question is of course positive, although to answer it we shall show that the set of surfaces where the spectrum does not have this property is Baire meagre. The answer to the third question is clearly yes if we allow deformations in the space of all Riemannian metrics, but we show that the answer is no if we restrict ourselves to metrics of fixed constant curvature.

The hypersurfaces we study are the nonempty subsets $\mathcal{E}(\alpha, \beta)$ of Teichmüller space where a pair of distinct simple closed geodesics $\alpha, \beta$ have the same length. When the intersection number $\iota(\alpha, \beta)$ is small, the surfaces $\mathcal{E}(\alpha, \beta)$ play an important part in the theory of fundamental domains for the mapping class group in low genus; see for instance Griffiths [15] and Maskit [20]. 
Our main theorem is the following:

Theorem 1.1 The set of surfaces with simple simple length spectrum is dense and its complement is Baire meagre. If $\mathcal{A}$ is a path in Teichmüller space $\mathcal{T}$ then there is a surface on $\mathcal{A}$ which has at least two distinct simple closed geodesics of the same length.

Let $\mathcal{E}$ denote the set of all surfaces with at least one pair of simple closed geodesics of equal length; $\mathcal{E}$ is the union of a countable family of nowhere dense subsets, namely the sets $\mathcal{E}(\alpha, \beta)$ where $\alpha, \beta$ vary over all distinct simple closed geodesics. The theorem asserts that $\mathcal{E}$ is dense and moreover that the complement contains no arcs and is thus totally disconnected. Our next point of interest is the topology of the sets $\mathcal{E}(\alpha, \beta)$ and $\mathcal{E}$.

Theorem 1.2 The sets $\mathcal{E}(\alpha, \beta)$ are connected analytic submanifolds of Teichmüller space. The set $\mathcal{E}$ is connected.

In the case of the Teichmüller space $\mathcal{T}_{1,1}^{\varepsilon}$ of a one-holed torus with fixed boundary length, a careful study of the asymptotic behaviour of the sets $\mathcal{E}(\alpha, \beta)$ will allow us to prove the following.

Theorem 1.3 Let $\alpha$ and $\beta$ be a pair of distinct simple closed geodesics on a one-holed torus. The set $\mathcal{E}(\alpha, \beta)$ is a simple path joining the points in the Thurston boundary of $\mathcal{T}_{1,1}^{\varepsilon}$ determined by the two unique simple closed geodesics with equal number of intersection points with both $\alpha$ and $\beta$.

We strongly suspect that the set of one-holed tori which contain three simple closed geodesics of equal length is nowhere dense.

Though the Euclidean torus is not negatively curved, it is useful to bear it in mind as a prototype. The reader is invited to check that our theorems above hold for Euclidean tori. Let $\mathcal{T}$ be the Teichmüller space of Euclidean tori, which one identifies with the upper half plane, or the $\tau$ plane, in the usual way. The rational $p / q \in \mathbb{R}$ corresponds to a simple curve of slope $p / q$ on the square torus which we shall use as a reference surface. The length of the curve on the surface corresponding to the parameter $\tau$ is $|p \tau+q|$ and geometry of the set $\mathcal{E}$ is readily understood in terms of elementary number theory. By computation, one sees that the set $\mathcal{E}(\alpha, \beta)$ is always a Poincaré geodesic, joining pairs of rationals on the real line. Reciprocally, the map $z \mapsto-\bar{z}$ preserves the rationals and fixes $[0, \infty]=\{\Re z=0\}$. For any rational $m / n$ the curves of slope $m / n$ and $-m / n$ have the same length at $\tau \in[0, \infty]$. One maps $[0, \infty]$ onto any geodesic $\left[a / b, a^{\prime} / b^{\prime}\right]$ using $\operatorname{PSL}(2, \mathbb{R})$, which is transitive on pairs of rationals and thus finds a 
pair of curves which are of equal length on $\left[a / b, a^{\prime} / b^{\prime}\right]$. The first sentence of Theorem 1.1 follows since quadratic irrationals are countable and the second because every pair of distinct points in the plane is separated by a Poincaré geodesic with rational endpoints. Unfortunately, in the more general situation we consider below, no such characterization of the sets $\mathcal{E}(\alpha, \beta)$ exists, although we obtain an analogous result for one holed hyperbolic tori with fixed boundary length in Theorem 1.3.

It is also interesting to note that the simple length spectrum of the square flat torus has unbounded multiplicity. The squares of the lengths are sums of squares of integers. By elementary number theory, for any sequence of integers $k_{n}$, each a product of $n$ distinct primes congruent to $1 \bmod 4$, the number of ways of writing $k_{n}$ as a sum as squares of coprime integers goes to infinity with $n$. It is well known that a prime $p \neq 2$ can be written as a sum of squares $x^{2}+y^{2}, x, y \in \mathbb{N}$ if and only if $p$ is congruent to 1 modulo 4 . It is also well known that there are infinitely many primes congruent to 1 modulo 4 . Such a prime $p$ admits a factorization $p=(x+i y)(x-i y)$ and $x+i y, x-i y$ are irreducible elements of the ring of Gaussian integers $\mathbb{Z}[i]$. Choose $n$ distinct primes $p_{k} \in \mathbb{N}, 1 \leq k \leq n$, let $a_{k} \in \mathbb{Z}[i]$ such that $p_{k}=a_{k} \bar{a}_{k}$ and let $N$ denote their product. It is immediate from the above that $N$ factorizes over $\mathbb{Z}[i]$ :

$$
N=\left(a_{1} \bar{a}_{1}\right)\left(a_{2} \bar{a}_{2}\right) \ldots\left(a_{n} \bar{a}_{n}\right) .
$$

Consider the set $R_{N}$ of Gaussian integers of the form $c_{1} c_{2} \ldots c_{n}$ where $c_{k} \in\left\{a_{k}, \bar{a}_{k}\right\}$. Note that the modulus squared of each element of $R_{n}$ is $N$. It is easy to check, using the fact that the Gaussian integers is a unique factorization domain, that $R_{N}$ contains exactly $2^{n-1}$ distinct elements. Note further that if $c_{1} c_{2} \ldots c_{n} \in R_{N}$ and $c_{1} c_{2} \ldots c_{n}=x+i y$ then $x, y$ are coprime integers, for otherwise there is a prime $p$ that divides $x, y$ hence $x+i y$ but this is impossible as the $c_{i}$ are irreducible and no two $c_{i}$ are complex conjugate.

Similarly, for the spectrum of lengths of all closed geodesics of hyperbolic surfaces, the multiplicities are unbounded. More precisely, Randol [23] shows (using results of Horowitz) that for any surface of constant curvature and any $n>0$, there is a set of $n$ distinct primitive geodesics of the same length. By the above, these curves necessarily have double points.

Finally, by the work of Schmutz-Schaller [28] and others on the systole, one has a lower bound on the multiplicity of the simple length spectrum as the surface varies over Teichmüller space. The existence (or not) of an upper bound for the multiplicity of the simple length spectrum for surfaces of a given signature is an open question. In particular, Schmutz-Schaller conjectured the following in [28] for once-punctured tori. 
Conjecture 1 The multiplicity of the simple length spectrum is bounded above by 6 for a once-punctured torus.

In fact, this conjecture is a geometric generalization of an equivalent version to the following well-known conjecture, as will be explained below.

Conjecture 2 (Markoff conjecture) The Markoff conjecture (see Frobenius [12]) is a conjecture in classical number theory. In its original form, it concerns solutions in the positive integers to the cubic

$$
x^{2}+y^{2}+z^{2}-x y z=0 .
$$

It states simply that if there are solutions $x \geq y \geq z$ and $x \geq y^{\prime} \geq z^{\prime}$ over the positive integers then $y=y^{\prime}$ and $z=z^{\prime}$.

At the time of writing, Conjecture 2 is known to be correct for $x$ a prime power; see Schmutz [27] and Button [7], and more recently Lang and Tan [19]. Other results related to this conjecture include Baragar [2] and Button [8].

Over the past few decades, several authors (see for instance Series [29], Haas [16], Schmidt and Sheingorn [26], Cohn [10;11] and Sarnak [25]) have had much success in translating problems in number theory into the language of surfaces and geodesics. Their efforts have in some cases provided a greater clarity to the understanding of some classical theorems, particularly in Diophantine approximation.

Let us outline the well-known correspondence between Markoff triples $(x, y, z)$ (solutions to the above problem) and triples of simple closed geodesics $(\alpha, \beta, \gamma)$ on a certain once-punctured torus (a torus with a single cusp as boundary).

There is, up to isometry, exactly one hyperbolic once-punctured torus, $\mathbf{M}$, with isometry group of order 12. This surface, commonly called the modular torus, is obtained as the quotient of the hyperbolic plane by a certain subgroup, $G$ of index 6 of the modular group $\operatorname{PSL}(2, \mathbb{Z})$ acting by linear fractional transformations. In fact $G$ is a normal subgroup of the modular group and the quotient group $S L(2, \mathbb{Z}) / G \simeq C_{2} \times S_{3}$ is isomorphic to the group of orientation preserving isometries of the quotient surface. By Riemann-Hurwitz, 6 is the maximum number of orientation preserving isometries of a once-punctured torus and, by a similar argument, 12 is the maximum number of isometries of a once-punctured torus.

If $(\alpha, \beta, \gamma)$ is a triple of simple geodesics on $\mathbf{M}$ which pairwise intersect in exactly one point (ie the three sets $\alpha \cap \beta, \beta \cap \gamma, \gamma \cap \alpha$ each consist of a single point) then

$$
\left(2 \cosh \frac{\ell(\alpha)}{2}, 2 \cosh \frac{\ell(\beta)}{2}, 2 \cosh \frac{\ell(\gamma)}{2}\right)
$$


is a solution to the cubic above. This is because one can find matrices $A, B, C=A B$ such that any pair generate $G$ and so that

$$
\operatorname{tr} A=2 \cosh \frac{\ell(\alpha)}{2}, \quad \operatorname{tr} B=2 \cosh \frac{\ell(\beta)}{2}, \quad \operatorname{tr} C=2 \cosh \frac{\ell(\gamma)}{2} ;
$$

the commutator of $A, B$ is parabolic, ie, has trace -2 , and, using the familiar trace identity in $S L(2, \mathbb{C})$ for the commutator (see Beardon [3]), one sees that these three numbers satisfy the cubic.

There is an equivalent conjecture to the Markoff conjecture which concerns simple closed geodesics on $\mathbf{M}$.

Conjecture 3 The modular torus $\mathbf{M}$ has the following property: if $\alpha, \beta$ are a pair of simple closed geodesics of the same length, then there is an isometry of $\mathbf{M}$ taking one to the other. We shall refer to this as the Markoff isometry property.

The equivalence of these conjectures can be shown as follows. We begin by noting that given 3 real numbers $x, y, z$ satisfying the cubic above there exist 3 matrices $A, B, A B=C \in S L(2, \mathbb{R})$ such that

$$
\operatorname{tr} A=x, \quad \operatorname{tr} B=y, \quad \operatorname{tr} C=z,
$$

and so that the commutator of $A, B$ is parabolic. Further, these matrices are unique up to conjugation in $S L(2, \mathbb{R})$ (see Goldman [14]).

Now let $\alpha, \alpha^{\prime}$ be a pair of simple geodesics on $M$ which have the same length. Since $\alpha$ (resp. $\left.\alpha^{\prime}\right)$ is simple there exist $A, B, C=A B \in G$ (resp. $A^{\prime}, B^{\prime}, C^{\prime}=A^{\prime} B^{\prime} \in G$ ) such that $A$ (resp. $A^{\prime}$ ) covers $\alpha$ (resp. $\alpha^{\prime}$ ), any two of $A, B, C$ (resp. $A^{\prime}, B^{\prime}, C^{\prime}$ ) generate $G$ and so that the trace of the commutator of $A, B$ (resp. $A^{\prime}, B^{\prime}$ ) is -2 . From the trace identity for this commutator, one sees that the quantities $\operatorname{tr} A, \operatorname{tr} B, \operatorname{tr} C$ and $\operatorname{tr} A^{\prime}, \operatorname{tr} B^{\prime}, \operatorname{tr} C^{\prime}$ satisfy the cubic. Since $\alpha$ and $\alpha^{\prime}$ have the same length $\operatorname{tr} A=\operatorname{tr} A^{\prime}$ so assuming the Markoff conjecture in its original form we must also have

$$
\operatorname{tr} B=\operatorname{tr} B^{\prime}, \quad \operatorname{tr} C=\operatorname{tr} C^{\prime} .
$$

By the uniqueness of $A, B, C$ in $S L(2, \mathbb{R})$ there exists a matrix $T \in S L(2, \mathbb{R})$ so that $T A T^{-1}=A^{\prime}, T B T^{-1}=B^{\prime}, T A T^{-1}=C^{\prime}$. Thus conjugation by $T$ leaves $G$ invariant and so this induces an isometry of $\mathbb{H} \backslash G$; since $T$ conjugates $A$ (which covers $\alpha$ ) to $A^{\prime}$ (which covers $\alpha^{\prime}$ ) this isometry takes $\alpha$ to $\alpha^{\prime}$ as required.

Now $\mathbf{M}$ is the only once-punctured torus which has isometry group $C_{2} \times S_{3}$, but not the only one-holed torus. (When the boundary of torus is a cusp or the boundary of the Nielsen core is a simple closed geodesic, we shall call it a one-holed torus.) Choose a real 
number $t \geq 3$; there are matrices, unique up to conjugacy in $S L(2, \mathbb{R}), A_{t}, B_{t}, A_{t} B_{t}=$ $C_{t} \in S L(2, \mathbb{R})$, each with trace $t$ and such that the trace of the commutator of $A_{t}, B_{t}$ is $3 t^{2}-t^{3}-2$. The group generated by $A_{t}, B_{t}$ is free, discrete and, when we think of it as acting on the upper half space by linear fractional transformations, the quotient surface, $M_{t}$, is homeomorphic to a torus with a point removed. When $t=3$ the trace of the commutator is -2 and the surface is the once-punctured torus $\mathbf{M}$. When $t>3$ the commutator is hyperbolic, the Nielsen core of the quotient is a proper subset and is bounded by a simple closed geodesic which is covered by the commutator. For any given $t \geq 3$, from the uniqueness of the matrices, $A_{t}, B_{t}, C_{t}$, it follows that the quotient surface has the isometry group specified.

In view of this observation, one is led to consider the following plausible generalization of the Markoff isometry property conjecture:

Conjecture 4 Let $\mathcal{T}_{1,1}^{\mathrm{S}}$ denote the set of hyperbolic one-holed tori which have isometry group isomorphic to $C_{2} \times S_{3}$. If $M$ is such a surface, then it enjoys the Markoff isometry property; that is if $\alpha, \beta$ are a pair of simple closed geodesics of the same length on $M$ then there is an isometry of this surface taking one to the other.

Notice that this conjecture is implied by a generalization to one-holed tori of SchmutzSchaller's Conjecture 1. By applying the analysis used to prove Theorem 1.1, we shall see that this conjecture is in fact false, and thus a generalized version of SchmutzSchaller's conjecture is also false. More precisely, we show the following.

Theorem 1.4 The subset of surfaces which fail to have the Markoff isometry property is dense in $\mathcal{T}_{1,1}^{S}$.

This means that the original Markoff conjecture is (probably) a conjecture in pure number theory and not tractable by hyperbolic geometry arguments.

Organization We begin by reviewing some basic facts concerning curves and geodesics from the theory of surfaces. Two preliminary results we readily use, Theorem 2.1 and Theorem 2.2, do not seem to be known in the form we require, and because the proofs are different in nature from the rest of the article, we defer them to Section 10. There then follows a discussion of the Dehn twist homeomorphism in relation to curves (Lemma 2). Using Dehn twists, we generate special sequences of geodesics and using these establish proposition Proposition 3.1, which is fundamental to our argument. This result, together with Theorem 2.2, implies Theorem 1.1. We then discuss why we believe that a careful modification of this argument provides evidence that the Markoff conjecture cannot be proved using techniques from Teichmüller theory. In 
the final section, we begin by showing that the hypersurfaces we study are connected submanifolds and that their union is also connected. Finally, a characterization of the hypersurfaces is given in the particular case of one-holed tori (with fixed boundary length) which is quite similar to the case of flat tori mentioned above.

Acknowledgments The first author would like to thank John Parker and Caroline Series for introducing him to the Markoff conjecture, Andy Haas for encouraging him to study the problem and David Epstein for guidance and helpful remarks. The second author would like to thank Benoit Bertrand, Thomas Gauglhofer and Alexander Lytchak for precious advice. Both authors would like to thank a variety of people they have bored with their unsuccessful geometric constructions while trying to solve the Markoff conjecture, including Gérard Maze and André Rocha. Finally, we are grateful to the referee for valuable remarks which have improved the paper.

\section{Preliminaries}

We review some elementary definitions and facts from the theory of surfaces; much of this is available in a more detailed treatment in either Abikoff [1], Beardon [3] or Buser [5]. Throughout $M$ will denote a surface with constant curvature -1 and we shall insist that $M$ is complete with respect to this metric (although we will only be concerned by what happens inside the convex core of the surface). This means that $M$ is locally modeled on the hyperbolic plane $\mathbb{H}^{2}$ and there is a natural covering map $\pi: \mathbb{H} \rightarrow M$. By $\mathcal{T}$ we mean the Teichmüller space of $M$, meaning the space of marked complete hyperbolic structures on $M$. The signature of a surface $M$ will be denoted $(g, n)$ where $M$ is homeomorphic to a surface of genus $g$ with $n$ simple closed boundary curves. If deemed necessary, we will denote $\mathcal{T}_{g, n}$ the Teichmüller space of surfaces of signature $(g, n)$. It is often useful to think of the Fenchel-Nielsen coordinates of Teichmüller space, namely the lengths of a pants decomposition of $M$ and a set of twist parameters which set how the interior simple closed geodesics (defined below) are pasted together. We insist on the fact the lengths of the boundary curves are allowed to vary.

Next one recalls some facts concerning curves on surfaces (see Buser [5] or Casson and Bleiler [9] for details). Firstly, a simple curve is a curve which has no self intersections. A curve is essential if it bounds neither a disc nor a punctured disc (annulus). For each free homotopy class which contains an essential simple loop, there is a unique geodesic representative. Further if such a free homotopy class contains a simple curve then this geodesic representative is also simple. Simple closed geodesics are called separating if they separate the surface into two connected components and nonseparating otherwise. 
Furthermore, a simple closed geodesic is called interior if it is entirely contained in the interior of the convex core of the surface.

There is a natural function, $\ell: \mathcal{T} \times$ essential homotopy classes $\rightarrow \mathbb{R}^{+}$, which takes the pair $M,[\alpha]$ to the length $\ell_{M}(\alpha)$ of the geodesic in the homotopy class $[\alpha]$ (measured in the Riemannian metric on $M$ ). It is an abuse, though common in the literature, to refer merely to the length of the geodesic $\alpha$ (rather than, more properly, the length of the geodesic in the appropriate homotopy class).

The set of simple closed geodesics on the surface $M$ is more than just an interesting curiosity. It was discovered by early investigators (Fricke et al) that the lengths of a carefully chosen finite subset of such curves could be used as a local coordinate system for the space of surfaces; these are often referred to as the moduli of the space. One way of seeing this is through the following theorem, which we shall not use explicitly although one could say that it contains the intuitive idea of how we shall proceed.

Theorem 2.1 Let $\mathcal{T}$ be the Teichmüller space of given signature. There is a fixed finite set of simple closed geodesics $\gamma_{1}, \ldots, \gamma_{n}$ such that the map

$$
\varphi: M \mapsto\left(\ell_{M}\left(\gamma_{1}\right), \ldots, \ell_{M}\left(\gamma_{n}\right)\right)
$$

is projectively injective on $\mathcal{T}$.

(A map $f: X \rightarrow V, V$ a real vector space is projectively injective if and only if $f(x)=t f(y)$, for some $t \in \mathbb{R}$, implies $x=y$.)

It is important to note that in the case where $\mathcal{T}$ is the Teichmüller space of a surface with boundary, the geodesics are allowed to be boundary geodesics. The nature of our investigation requires us to study interior simple closed geodesics, so we need a generalization of Theorem 2.1 where the set of simple closed geodesics $\gamma_{1}, \ldots, \gamma_{n}$ are all interior. While concocting a theorem suitable for our needs, we discovered that the generalization to surfaces with boundary, under the assumption that the geodesics are all interior, is false. What is true however is the following theorem.

Theorem 2.2 Let $\mathcal{T}$ be the Teichmüller space of given signature. Then there is a set $\Gamma:=\left\{\gamma_{1}, \ldots, \gamma_{n}\right\}$ of interior simple closed curves such that for any given point in $M \in \mathcal{T}$, there are a finite many $M^{\prime} \in \mathcal{T}$ such that $\lambda\left(\ell_{M}\left(\gamma_{1}\right), \ldots, \ell_{M}\left(\gamma_{n}\right)\right)=$ $\left(\ell_{M^{\prime}}\left(\gamma_{1}\right), \ldots, \ell_{M^{\prime}}\left(\gamma_{n}\right)\right)$ for some $\lambda \in \mathbb{R}$.

We give proofs of both Theorem 2.1 and Theorem 2.2, but because these are different in nature to the rest of the article, their proofs are deferred to Section 10. In Section 10, examples are given to highlight the differences between Theorem 2.1 and Theorem 2.2. 
In particular, Example A.1 shows that a generalization of Theorem 2.1 is false for four holed spheres. Note that Theorem 2.1 is well known to specialists, and for closed surfaces is implicit in Thurston [30].

Of particular use to us is the following reformulation of Theorem 2.2.

Theorem 2.3 Let $\mathcal{T}$ be the Teichmüller space of given signature, and $\Gamma$ be the set of curves of Theorem 2.2. For any $M \in \mathcal{T}$, there are finitely many $M^{\prime} \in \mathcal{T}$ such that

$$
\frac{\ell_{M^{\prime}}(\alpha)}{\ell_{M^{\prime}}(\beta)}=\frac{\ell_{M}(\alpha)}{\ell_{M}(\beta)}
$$

for all $\alpha, \beta \in \Gamma$.

If we consider only closed surfaces then the following argument proves a slightly stronger result than the theorem stated above.

For the argument we use Dehn twists : a Dehn twist $\mathbf{T}_{\alpha}: M \mapsto M$ around a simple closed curve $\alpha$ is a homeomorphism (defined up to isotopy) of the surface $M$ to itself which is the identity on the complement of a regular neighborhood of $\alpha$. The Dehn twist takes a geodesic $\gamma$ meeting $\alpha$ to a (possibly nongeodesic) curve. However, since this curve is also essential, we can straighten it, ie, take the unique closed geodesic in the homotopy class of $\mathbf{T}_{\alpha}(\gamma)$. Since the twist is a homeomorphism, the resulting geodesic is simple if and only if the original geodesic $\gamma$ was. By convention, we say that a Dehn twist takes simple closed geodesics to simple closed geodesics.

Now let $A, B$ be points in a Teichmüller space of a closed hyperbolic surface; we now give a sketch of the existence of simple closed curves $\alpha, \beta$ such that

$$
\frac{\ell_{A}(\alpha)}{\ell_{A}(\beta)} \neq \frac{\ell_{B}(\alpha)}{\ell_{B}(\beta)}
$$

We shall suppose that there exists a simple closed curve $\gamma$ such that $A, B$ lie are in the same orbit for Fenchel-Nielsen twist along $\gamma$; by replacing $\gamma$ by a measured lamination and using Thurston's earthquake theorem one obtains the general case. Suppose further that $\gamma$ is non separating then there is a simple closed geodesic $\gamma^{\prime}$ that meets $\gamma$ in exactly one point. In fact, if $\mathbf{T}_{\gamma}^{n}\left(\gamma^{\prime}\right)$ denotes the simple geodesic freely homotopic to the image of $\gamma^{\prime}$ by the (right) Dehn twist along $\gamma$ iterated $n$ times, then $\mathbf{T}_{\gamma}^{n}\left(\gamma^{\prime}\right)$ meets $\gamma$ in exactly one point too. Let $\theta_{n}$ denote the signed angle at $\mathbf{T}_{\gamma}^{n}\left(\gamma^{\prime}\right) \cap \gamma$, then it is not hard to show, using hyperbolic trigonometry, that $\theta_{n}$ is monotone in $n$, and furthermore that for any $\epsilon>0$ there exists $N$ such that

$$
\cos \left(\theta_{-N}\right)<-1+\epsilon, \quad \cos \left(\theta_{N}\right)>1-\epsilon .
$$


Take $\alpha=\mathbf{T}_{\gamma}^{N}\left(\gamma^{\prime}\right)$ and $\beta=\mathbf{T}_{\gamma}^{N}\left(\gamma^{\prime}\right)$. Following Kerckhoff [18], we see that as we move from $A$ to $B$ along the Fenchel-Nielsen twist orbit, $\ell(\alpha)$ is monotone decreasing whilst $\ell_{\beta}$ is monotone increasing and

$$
\frac{\ell_{A}(\alpha)}{\ell_{A}(\beta)}<\frac{\ell_{B}(\alpha)}{\ell_{B}(\beta)}
$$

Finally, with respect to the differential structure of Teichmüller space, we have the following:

Proposition 2.4 For each closed geodesic $\alpha$, the function $M \mapsto \ell_{M}(\alpha)$ is an analytic function.

\section{Dehn Twists and length ratios}

The proof of our main Theorem 1.1 is based on a trick involving the topological manipulation of curves. In particular, we shall generate a sequence of simple closed curves with a certain prescribed property which is outlined below (basically their lengths should satisfy part 2 of Lemma 2).

A simple topological argument, pasting together curves, establishes the next lemma (left to the reader as an exercise.)

Lemma 1 Let $M$ be a surface and $\alpha$ a simple closed geodesic on $M$. If $\alpha$ is not a boundary geodesic then there is a simple closed geodesic $\alpha_{0}$ which meets $\alpha$ in exactly 2 points.

Remark 3.1 We work with pairs of curves which meet in two points because a simple closed geodesic which divides the surface into 2 components meets any other geodesic loop in at least 2 points. A surface for which every non peripheral simple closed geodesic is separating is called a planar surface. Topologically, planar surfaces are $n$-holed spheres. The one-holed torus is not a planar surface, and in fact it is the only topological type of surface where every simple closed geodesic is non separating. By choosing a pair of curves as in the lemma above, we are able to treat the case of planar surfaces without much extra work.

Using Dehn twists around $\alpha$, we shall construct a sequence of geodesics with an interesting sequence of lengths. We construct the sequence inductively as follows: let $\alpha_{0}$ be any geodesic which meets $\alpha$ in exactly two points as in Lemma 1 . Then let $\alpha_{n}$ be the right Dehn twist of $\alpha_{n-1}$ round $\alpha$. This gives us a well-defined sequence of curves $\left\{\alpha_{n}\right\}_{n=0}^{\infty}$, each of which is closed and simple. 
Lemma 2 With $\alpha,\left\{\alpha_{k}\right\}_{k=0}^{\infty}$ as above:

(1) For each $k=1, \ldots \infty$ the curve $\alpha_{k}$ meets $\alpha$ in 2 points and each $\alpha_{k}$ is simple.

(2) For all $k \in \mathbb{N}$,

$$
2 k \ell(\alpha)-\ell\left(\alpha_{0}\right)<\ell\left(\alpha_{k}\right)<2 k \ell(\alpha)+\ell\left(\alpha_{0}\right) .
$$

Proof The first claim is immediate since homeomorphism takes simple curves to simple curves. The second is a consequence of the triangle inequality as can be seen in Figure 1 where the diagonal arc represents a lift of the curve $\alpha_{k}$. The vertical segments

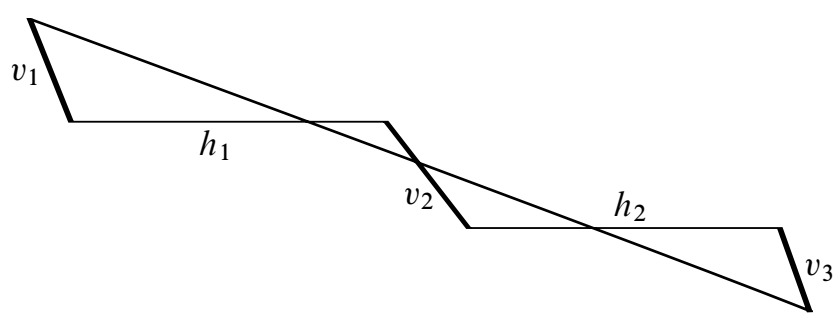

Figure 1

$v_{1}, v_{2}, v_{3}$ correspond to the lifts of three arcs of $\alpha_{0}$ and $\ell\left(v_{1}\right)+\ell\left(v_{2}\right)+\ell\left(v_{3}\right)=\ell\left(\alpha_{0}\right)$. The horizontal segments $h_{1}, h_{2}$ are lifts of two arcs of the (nonprimitive) curve $n \alpha$ with $\ell\left(h_{1}\right)+\ell\left(h_{2}\right)=n \ell(\alpha)$. By applying the triangle inequality to the different triangles one obtains the two inequalities.

We note that, by using Riemannian comparison theorems, this result is true even when $M$ does not have constant curvature but merely pinched negative curvature.

Fix a surface $M$ and let $\alpha, \beta$ be distinct simple closed geodesics. Our aim is to calculate the ratio $\ell(\alpha) / \ell(\beta)$ from the asymptotic formula in Lemma 2. For $\alpha$, resp. $\beta$, choose $\alpha_{0}$, resp. $\beta_{0}$, as in Lemma 1. Define $\alpha_{j}:=\mathbf{T}_{\alpha}^{j}\left(\alpha_{0}\right)$, resp. $\left.\beta_{j}:=\mathbf{T}_{\beta}^{j}\left(\beta_{0}\right)\right)$. Set $B_{i}:=\left\{\beta_{k}: \ell\left(\beta_{k}\right) \leq \ell\left(\alpha_{i}\right)\right\}$.

Proposition 3.1 With the notation above:

$$
\frac{\sharp B_{i}}{i} \longrightarrow \frac{\ell(\alpha)}{\ell(\beta)} .
$$


Proof By Lemma 2 we have

$$
\sharp B_{i} \leq \sharp\left\{k: 2 k \ell(\beta)-\ell\left(\beta_{0}\right) \leq 2 i \ell(\alpha)+\ell\left(\alpha_{0}\right)\right\},
$$

and

$$
\sharp B_{i} \geq \sharp\left\{k: 2 k \ell(\beta)+\ell\left(\beta_{0}\right) \leq 2 i \ell(\alpha)-\ell\left(\alpha_{0}\right)\right\} \text {. }
$$

It follows that

$$
i \frac{\ell(\alpha)}{\ell(\beta)}-\frac{\ell\left(\beta_{0}\right)}{2 \ell(\beta)}-\frac{\ell\left(\alpha_{0}\right)}{2 \ell(\beta)} \leq \sharp B_{i} \leq i \frac{\ell(\alpha)}{\ell(\beta)}+\frac{\ell\left(\alpha_{0}\right)}{2 \ell(\beta)}+\frac{\ell\left(\beta_{0}\right)}{2 \ell(\beta)} .
$$

The statement of the proposition is immediate.

In the sequel, we shall denote $N(M)_{i}:=\sharp B_{i}$ for a given choice of $M$.

\section{Surfaces with a pair of geodesics of equal length}

For completeness we give a brief account of the nature of the sets $\mathcal{E}(\alpha, \beta)$ in terms of elementary pointset topology. The results presented in this section seem to be well known but do not appear in the literature.

It should be possible to develop a very precise theory of these sets in terms of algebraic functions, via Fricke trace calculus, however, we will not consider that approach further here.

The next result exploits the fact, by Proposition 2.4, that the function $M \mapsto \ell_{M}(\alpha)-$ $\ell_{M}(\beta)$ is analytic.

Lemma 3 Let $\alpha, \beta$ be a pair of simple closed geodesics.

(1) $\mathcal{E}(\alpha, \beta)$ is non empty.

(2) $\mathcal{E}(\alpha, \beta)$ is a closed subset with no interior, ie, its complement is open dense in $\mathcal{T}$.

(3) Let $\mathcal{E}=\bigcup \mathcal{E}(\alpha, \beta)$ where the union is taken over all pairs of simple closed geodesics and set $\mathcal{N} e q=\mathcal{T} \backslash \mathcal{E}$. Then $\mathcal{N} e q$ is dense.

Proof It is easy to show that, for each pair of simple closed geodesics $\alpha \neq \beta, \mathcal{E}(\alpha, \beta)$ is closed. This follows when one notes that $\mathcal{E}(\alpha, \beta)$ is the zero set for the continuous function $M \mapsto f_{\alpha, \beta}(M):=\ell_{M}(\alpha)-\ell_{M}(\beta)$.

Next one establishes that $\mathcal{E}(\alpha, \beta)$ has no interior, ie, contains no open sets. Recall that if a real analytic function is constant on an open set in its domain then in fact it is constant on the entire component which contains this set. We shall apply this to our 
function $f_{\alpha, \beta}$ to establish the claim. Suppose that $\alpha$ and $\beta$ are simple geodesics and they have the same length on some open subset of $\mathcal{T}$ (so that the function above is zero on this open set). Since $\mathcal{T}$ is connected, the function is zero over the whole space, ie, the corresponding geodesics must have the same length over the whole space. If $\alpha$ and $\beta$ are distinct then it is easy to find a sequence of deformations of the surface such that the length of $\alpha$ remains bounded while the length of $\beta$ tends to infinity (for example, if $\alpha$ meets $\beta$ the right Dehn twist round $\alpha$ provides such a sequence). Thus $\alpha=\beta$.

We show that $\mathcal{E}(\alpha, \beta)$ is non empty by applying the intermediate value theorem to our function $f_{\alpha, \beta}$ on Teichmüller space, which is connected. The claim is true if there are surfaces $X$ and $Y$ such that on $X$, the curve $\alpha$ is very short and $\beta$ is very long, and on $Y \beta$ is very short and $\alpha$ is very long. There are two cases to consider depending on whether $\alpha$ and $\beta$ intersect or not. Suppose that $\alpha$ and $\beta$ do not meet; then there is a pants decomposition of $M$ so that both $\alpha$ and $\beta$ are curves in the decomposition. This enables one to find the surfaces $X, Y$ quite easily, since one can specify the lengths of the curves in a pants decomposition independently. On the other hand, if $\alpha, \beta$ meet, then by the collar theorem [5], for any $M \in \mathcal{T}$, their lengths satisfy the inequalities

$$
\begin{aligned}
& \ell_{M}(\alpha) \geq 2 \operatorname{arcsinh}\left(\frac{1}{\sinh \frac{\ell_{M}(\beta)}{2}}\right), \\
& \ell_{M}(\beta) \geq 2 \operatorname{arcsinh}\left(\frac{1}{\sinh \frac{\ell_{M}(\alpha)}{2}}\right) .
\end{aligned}
$$

Now by choosing a surface $X$, resp. $Y$, where the length of $\alpha$ is indeed very small, resp. where the length of $\beta$ is very small, we are guaranteed that the length of $\beta$, resp. $\alpha$, is very long.

One notes that the final part is just the result of the Baire category theorem applied to the sets $\mathcal{T} \backslash \mathcal{E}(\alpha, \beta)$. This completes the proof.

In some sense the complement of $\mathcal{E}, \mathcal{N} e q$, is a big set (Baire). Another convenient way of thinking of this is that for the generic surface in Teichmüller space, the length map from the set of simple geodesics to the positive reals is injective. It is also interesting to contrast the above lemma with the following observation due to Randol [23]: there are pairs of closed geodesics $\alpha \neq \beta$ which have the same length over the whole of Teichmüller space. By the above, these geodesics necessarily have self intersections.

Lemma 3 proves the first part of Theorem 1.1, and the remaining part is the goal of the next section. 


\section{Total disconnectedness}

In this section we establish that $\mathcal{N} e q$, the complement of $\mathcal{E}$ in Teichmüller space $\mathcal{T}$ is totally disconnected ie it contains no nontrivial arc. An arc is the continuous image of the closed unit interval, an arc is trivial if it is a constant map on the interval; obviously an arc is nontrivial if and only if it contains a subarc with distinct endpoints. Our proof consists of applying the intermediate value theorem to a certain continuous function which we concoct using Proposition 3.1.

Let $\mathcal{A}$ be a nontrivial arc in Teichmüller space. By Theorem 2.3, there exist distinct points $X, Y \in \mathcal{A}$ and a pair of simple closed geodesics $\alpha, \beta$ such that

$$
\ell_{X}(\alpha) / \ell_{X}(\beta) \neq \ell_{Y}(\alpha) / \ell_{Y}(\beta) .
$$

Now by Proposition 3.1, we see that there is an integer $i$ such that the numbers $N(X)_{i}, N(Y)_{i}$, defined in Section 3, are different. In particular, there are simple closed geodesics $\alpha_{i}$ and $\beta_{n}$ such that

and

$$
\begin{aligned}
& \ell_{X}\left(\beta_{n}\right)>\ell_{X}\left(\alpha_{i}\right) \\
& \ell_{Y}\left(\beta_{n}\right)<\ell_{Y}\left(\alpha_{i}\right) .
\end{aligned}
$$

Now $M \mapsto \ell_{M}\left(\alpha_{i}\right)-\ell_{M}\left(\beta_{n}\right)$ is a continuous function, so applying the intermediate value theorem to $\mathcal{A}$, between the points $X$ and $Y$, yields the existence of a surface $Z \in \mathcal{A}$ so that $\ell_{Z}\left(\alpha_{i}\right)=\ell_{Z}\left(\beta_{n}\right)$.

\section{The order in lengths of simple closed geodesics determines a point in Teichmüller space}

The purpose of this section is to observe that our analysis has produced a curious fact: for closed surfaces (or surfaces with fixed boundary length) and one-holed tori, the orders between lengths of simple closed geodesics determine a point in Teichmüller space. To see this, recall that one of the main ingredients in our proof of Theorem 1.1 is that, in many cases, including the case of surfaces with fixed boundary lengths and in particular closed surfaces or surfaces with cusp boundary (Theorem 2.1) and one-holed tori (Lemma 11 in Section 10), the map from Teichmüller space to the marked interior simple length spectrum is projectively injective. When this is the case, we've shown that between any two distinct points of Teichmüller space there is a pair of simple closed geodesics $\alpha$ and $\beta$ such that their order in length is reversed. From this observation, one obtains the following result. 
Theorem 6.1 Let $\mathcal{T}$ be the Teichmüller space of a surface with given boundary length or of a one-holed torus with variable boundary length. Then the map that sends a point in $\mathcal{T}$ to the relative orders in length between simple closed geodesics is injective.

Example A.1 of two nonisometric four-holed spheres with identical interior marked simple length spectrum at the end of Section 10 shows that one must be very careful when trying to generalize the above theorem to arbitrary signature with variable boundary lengths.

\section{Application to the Markoff conjecture}

Recall that $\mathcal{T}_{1,1}^{\mathrm{S}}$ is the set of all one-holed tori with maximal isometry group. The mapping class group of acts transitively on connected components of $\mathcal{T}_{1,1}^{\mathrm{s}}$ each of which is a smooth 1-dimensional submanifold of the Teichmüller space of one-holed tori $\mathcal{T}_{1,1}$. We now apply our analysis of multiplicities to this subset.

Theorem 7.1 The subset of $\mathcal{T}_{1,1}^{S}$ which does not have the Markoff isometry property is dense.

Remark 7.1 For the same reason as was given for the set $\mathcal{E} \subset \mathcal{T}$ in Lemma 3, the subset consisting of those surfaces $M_{t}$ not having the Markoff isometry property is a meager set in the sense of Baire. To do this one needs to see that, for $\alpha$ and $\beta$ not related by an isometry, the set $\mathcal{E}(\alpha, \beta)$ meets a connected component of $\mathcal{T}_{1,1}^{\mathrm{s}}$ in a finite number of points. This can be done presenting $\mathcal{E}(\alpha, \beta)$ and the connected component of $\mathcal{T}_{1,1}^{\mathrm{S}}$ as algebraic subsets of $\mathbb{R}^{3}$ using the Fricke trace calculus.

Proof Consider $X$ and $Y$, two distinct points in some connected component of $\mathcal{T}_{1,1}^{\mathrm{s}}$. Since $\mathcal{T}_{1,1}^{\mathrm{s}}$ is a submanifold of $\mathcal{T}_{1,1}$, it follows that there is an arc $\mathcal{A} \subset \mathcal{T}$ between $X$ and $Y$. By the results of Section 5, there is a pair of geodesics $(\alpha, \beta)$ such that $\ell_{X}(\alpha)<\ell_{X}(\beta)$ and $\ell_{Y}(\beta)<\ell_{Y}(\alpha)$, and thus a surface $Z \in \mathcal{A}$ such that $\ell_{Z}(\alpha)=\ell_{Z}(\beta)$. Since the connected component of $\mathcal{T}_{1,1}^{\mathrm{s}}$ is an embedded line no two surfaces $X, Y$ in the connected component are related by an element of the mapping class group (ie a change of marking). This means that if there is a surface $X$ in the connected component such that $\ell_{X}(\alpha) \neq \ell_{X}(\beta)$ then there is no surface $X^{\prime}$ (in the connected component) such that an isometry of $X^{\prime}$ takes $\alpha$ to $\beta$. In particular the surface $Z$ constructed above does not have the Markoff isometry property. This establishes the claim of the theorem. 
Remark 7.2 The surface $Z$ in the proof of the theorem is a one-holed torus with 12 distinct simple closed geodesics of equal length. As mentioned in the introduction, an upper bound on the number of simple closed geodesics of equal length on a one-holed torus would have to be at least 12 .

\section{Topology of $\mathcal{E}(\alpha, \beta)$}

In this section we prove Theorem 1.2 which tells us that our hypersurfaces are connected submanifolds and that their reunion is also connected. Let us begin by showing that they are submanifolds.

Lemma $4 \mathcal{E}(\alpha, \beta)$ is a smooth codimension 1 submanifold.

Proof This follows from the implicit function theorem, a surface $M \in \mathcal{E}$ is a regular point for the function $M \mapsto \ell_{M}(\alpha)-\ell_{M}(\beta)$ if

$$
d \ell_{M}(\alpha)-d \ell_{M}(\beta) \neq 0 \text {. }
$$

It suffices to find a single (smooth) deformation $\phi_{t}, \phi_{0}=M$ such that

$$
d \ell_{M}(\alpha) \cdot \dot{\phi}_{t} \neq d \ell_{M}(\beta) \cdot \dot{\phi}_{t},
$$

where $\dot{\phi}_{t}=\left.\frac{d \phi_{t}}{d t}\right|_{t=0}$. In fact since $\ell_{M}(\alpha)=d \ell_{M}(\beta)$ it suffices to find a deformation $\phi_{t}$ such that

$$
\frac{d \ell_{M}(\alpha)}{\ell_{M}(\alpha)} \cdot \dot{\phi}_{t} \neq \frac{d \ell_{M}(\beta)}{\ell_{M}(\beta)} \cdot \dot{\phi}_{t} .
$$

We explain why Thurston's stretch maps provide $\phi_{t}$ satisfying this latter condition. Stretch maps were introduced by Thurston in [30] as maps minimising the bi-Lipschitz constant between pairs of hyperbolic structures on the same closed surface. A stretch map $\phi_{t}$ is constructed from a maximal geodesic lamination $\lambda$; recall that a geodesic lamination is maximal if and only every complementary region is either an ideal triangle or a punctured monogon. One constructs a (partial) foliation of complementary regions by horocyclic segments. The hyperbolic metric induces a transverse Holder distribution on this foliation. Roughly speaking, the stretch map consists of multiplying this transverse distribution by $e^{t}$. This yields a hyperbolic structure such that the length of the maximal measured sublamination of $\lambda$ is also multiplied by $e^{t}$.

To show connectedness, we will need the following lemma which concerns spaces homeomorphic to $\mathbb{R}^{n}$ (such as Teichmüller space). 
Lemma 5 Let $U, V$ be open connected sets of $\mathbb{R}^{n}$ such that $\bar{U} \cap \bar{V}=\partial U=\partial V$ and $\bar{U} \cup V=\mathbb{R}^{n}$. Then $\partial U=\partial V$ is connected.

Proof Essentially this lemma follows from the fact that $\mathbb{R}^{n}$ has the Phragmen-Brouwer Property [4] which is outlined below. A topological space is said to have the PhragmenBrouwer property if, for two given disjoint closed sets $D$ and $E$ and two points $a$ and $b$, neither $D$ nor $E$ separates $a$ and $b$, then $D \cup E$ does not separate $a$ and $b$. (A set separates two points if the two points lie on distinct connected components of the complement of the set.)

Denote $F=\partial U=\partial V$ and suppose $F$ is not connected, ie, $F=F_{1} \cup F_{2}$ where $F_{1}$ and $F_{2}$ are disjoint nonempty closed sets. Now clearly, because $\mathbb{R}^{n}$ has the PhragmenBrouwer property, either the complement of $F_{1}$ or $F_{2}$ is disconnected. Suppose then that $F_{1}^{c}=A \cup B$ where $A$ and $B$ are disjoint and open. Now $U \subset A \cup B$ is connected thus we can suppose that $U \subset A$. Also, $F_{2} \subset A \cup B$ is closed and a subset of $\partial A$, so $F_{2} \subset A$. The set $V \subset A \cup B$ is also connected and $\partial V \cap A \supset F_{2} \neq \varnothing$ so $V \subset A$. Now $\bar{A}=\mathbb{R}^{n}$ and $B=\varnothing$, a contradiction.

Using this lemma and stretch maps we can show the following:

Lemma 6 The hypersurfaces $\mathcal{E}(\alpha, \beta)$ are connected.

Proof If $\alpha$ and $\beta$ are disjoint, then they can be decomposed into a pants decomposition and because Fenchel-Nielsen parameters determine both the points and the topology of Teichmüller space, the result is obvious.

Now for intersecting $\alpha$ and $\beta$, denote by $\mathcal{E}^{+}$, resp. $\mathcal{E}^{-}$, the open subsets of $\mathcal{T}$ where $\ell(\alpha)>\ell(\beta)$, resp. where $\ell(\alpha)<\ell(\beta)$. We begin by showing that these subsets of $\mathcal{T}$ are connected. Let $M_{1}, M_{2} \in \mathcal{E}^{+}$. Using a stretch map, it is possible to continuously deform a surface such that a given simple curve's length is decreased and more so than any other simple curve. Furthermore, its length will go to 0. Applying a stretch map to $\beta$ on both $M_{1}$ and $M_{2}$, we can find two finite paths in Teichmüller space $c_{1}, c_{2}$ such that $c_{1}(0)=M_{1}, c_{2}(0)=M_{2}$ and $c_{1}(1)=M_{1}^{\prime}, c_{2}(1)=M_{2}^{\prime}$ where $\ell_{M_{1}^{\prime}}(\beta), \ell_{M_{2}^{\prime}}(\beta)<2 \operatorname{arcsinh} 1$. Because of the property of stretch maps, these paths remain entirely in $\mathcal{E}^{+}$. By looking at Fenchel-Nielsen coordinates which include the length of $\beta$, it is easy to see that the subset of $\mathcal{T}$ such that $\ell(\beta)<2 \operatorname{arcsinh} 1$ is connected. Note that by the collar theorem and the fact that $\alpha$ and $\beta$ intersect, any surface with $\ell(\beta)<2 \operatorname{arcsinh} 1$ also has $\ell(\alpha)>2 \operatorname{arcsinh} 1$, and thus is contained in $\mathcal{E}^{+}$. This shows that $\mathcal{E}^{+}$is connected. Using the same argument, one shows that $\mathcal{E}^{-}$ is connected. 
Applying Lemma 5 to $U:=\mathcal{E}^{+}$and $V:=\mathcal{E}^{-}$(and of course $\mathcal{E}(\alpha, \beta)=\partial \mathcal{E}^{+}=\partial \mathcal{E}^{-}$), we obtain the result.

We now need to show that the reunion of the hypersurfaces is connected which follows from the fact that $\mathcal{N} e q$ is totally disconnected and the following lemma.

Lemma 7 Let $N$ be a totally disconnected set of $\mathbb{R}^{n}$ with $n>2$. Then $\mathbb{R}^{n} \backslash N$ is connected.

Proof $\mathbb{R}^{n}$ is a Cantor manifold (a result due to Urysohn for $n=3$ and to Aleksandrov for $n>3$ ), meaning that a closed subset that separates has topological dimension at least $n-1$. Of course, a totally disconnected subset $N$ is not (necessarily) closed. However, if $N$ separates then it contains a closed subset that separates as follows. Let $M$ be the complement of $N$ that is not connected, thus is equal to $(A \cap M) \cup(B \cap M)$ where $A$ and $B$ are open nonempty sets which are disjoint on $M$. Thus $A \cap B \subset N$. But as $\mathrm{N}$ is totally disconnected, $A \cap B=\varnothing$. Now the complement of $A \cup B$, say $F$, is closed, separates $\mathbb{R}^{n}$, and is contained in $N$. But its topological dimension cannot be greater than 0 because $N$ has topological dimension 0 , and thus cannot separate, a contradiction.

\section{Asymptotic behaviour of $\mathcal{E}(\alpha, \beta)$}

The goal of this section is to prove Theorem 9.1 which gives, in the particular case of a one-holed torus with fixed boundary length, a "coarse" description of $\mathcal{E}(\alpha, \beta)$. We show that $\mathcal{E}(\alpha, \beta)$ has two ends and that these determine a pair of points in the Thurston boundary of Teichmüller space. In fact these points are the same pair of geodesics as in the case of flat tori outlined in the introduction. Our point of departure is the observation that, by the collar lemma, one expects that if $M \in \mathcal{E}(\alpha, \beta)$ contains a (very) short essential simple closed geodesic $\gamma$ then

$$
\iota(\gamma, \alpha)=\iota(\gamma, \beta)
$$

We begin by studying this equation.

\subsection{Curves and homology}

Let $\mathcal{T}_{1,1}^{\varepsilon}$ denote the Teichmüller space of one-holed tori with some fixed boundary length $\varepsilon$. Let $\gamma$ be an oriented primitive essential simple closed geodesic and let $(t, \ell)$ be Fenchel-Nielsen parameters for this choice of simple closed curve. Associated to $\gamma$ is a foliation of $\mathcal{T}_{1,1}^{\varepsilon}$ by level sets of $\ell_{M}(\gamma)=\ell$ for $M \in \mathcal{T}_{1,1}^{\varepsilon}$. We make the 
convention that if a curve is a $k$ iterate of a primitive curve, then each intersection point (geometric or algebraic) is counted $k$ times.

Let us briefly recall some facts about homology classes and oriented simple closed curves. Write $[\gamma] \in H_{1}(M, \mathbb{Z})$ for the integer homology class determined by a simple closed curve $\gamma$ and $\iota_{a}(\cdot, \cdot)$ for the algebraic intersection number (relative to a fixed orientation of the surface). There is an essential simple closed curve $\gamma^{\prime}$ which meets $\gamma$ just once and, after possibly changing the orientation, we may suppose that $\iota_{a}\left(\gamma, \gamma^{\prime}\right)=1$. We say that the pair $\left([\gamma],\left[\gamma^{\prime}\right]\right)$ form a canonical basis for homology. The intersection numbers $\iota_{a}(\gamma, \alpha)$ and $\iota_{a}(\delta, \alpha)$ define a unique oriented simple closed curve up to isotopy. To each pair $(k, l) \in \mathbb{Z} \times \mathbb{Z}$, we associate the unique oriented (not necessarily primitive) simple closed geodesic $[\alpha]=k[\gamma]+l\left[\gamma^{\prime}\right]$ where $k=\iota_{a}\left(\alpha, \gamma^{\prime}\right)$ and $l=-\iota_{a}(\alpha, \gamma)$. Recall that, in the particular case of the one-holed torus, two simple closed curves are homologous if and only if they are isotopic. The following equation relating algebraic and geometric intersection numbers will of use in studying equation (9-1):

$$
\left|\iota_{a}(\alpha, \beta)\right|=\iota(\alpha, \beta) .
$$

Geometrically this means that a pair of oriented simple closed geodesics $\alpha, \beta$ on a torus always intersect "in the same way", ie, there are no arcs of $\alpha$ that leave $\beta$ and come back to $\beta$ on the same side of $\beta$. Essentially, equation (9-2) holds because on a one-holed torus no pair of arcs of simple closed geodesics form a bigon nor even a bigon surrounding the boundary curve of the torus $[6 ; 16]$.

Lemma 8 If $\gamma_{1}$ is an essential simple closed geodesic such that $\iota\left(\alpha, \gamma_{1}\right)=\iota\left(\beta, \gamma_{1}\right)$, then $\pm\left[\gamma_{1}\right]=[\alpha] \pm[\beta]$.

Proof Let $[\gamma],\left[\gamma^{\prime}\right]$ be a basis of the integer homology, then there exists $a, b, c, d \in \mathbb{Z}$ such that $[\alpha]=a[\gamma]+b\left[\gamma^{\prime}\right]$ and $[\beta]=c[\gamma]+d\left[\gamma^{\prime}\right]$ with $a d-b c \neq 0$. Suppose $\left[\gamma_{1}\right]=x[\gamma]+y\left[\gamma^{\prime}\right]$ with $x, y \in \mathbb{Z}^{2} \backslash\{0,0\}$ then it satisfies

$$
\iota\left(\gamma_{1}, \alpha\right)=|a y-x b|=|c y-d x|=\iota\left(\gamma_{1}, \beta\right),
$$

since geometric intersection number is just the absolute value of the algebraic intersection number. Dropping absolute values,

$$
a y-b x= \pm(c y-d x),
$$

that is $(x, y)$ is a solution of one of the following:

$$
\begin{gathered}
(a+c) y-(b+d) x=0, \\
(a-c) y-(b-d) x=0 .
\end{gathered}
$$


The first equation gives $\pm\left[\gamma_{1}\right]=[\alpha]+[\beta]$ and the second $\pm\left[\gamma_{1}\right]=[\alpha]-[\beta]$.

\subsection{Existence}

Given a pair of primitive simple closed geodesics $\alpha$ and $\beta$, the preceding lemma yields a pair of primitive essential simple closed geodesics such that the geometric intersection number with both $\alpha$ and $\beta$ is the same. We will now show that the ends of $\mathcal{E}(\alpha, \beta)$ meet the Thurston boundary in these points Let $\gamma, \gamma^{\prime}$ be a pair of simple closed geodesic such that $\left([\gamma],\left[\gamma^{\prime}\right]\right)$ is a canonical homology basis. Thus $[\alpha]=a\left[\gamma^{\prime}\right] \pm n[\gamma]$ and $[\beta]=b\left[\gamma^{\prime}\right] \pm n[\gamma]$. After possibly changing orientations of the curves $\alpha$ and $\beta$, we may suppose that $[\alpha]=a[\gamma]-n\left[\gamma^{\prime}\right]$ and $[\beta]=b[\gamma]-n\left[\gamma^{\prime}\right]$.

For any given torus, in [21] it is shown that $[\gamma] \mapsto \ell(\gamma)$ extends to a norm, equal in fact to the stable norm, on the first homology group of the torus $H_{1}(T, \mathbb{R})$. The unit ball of a norm is a convex set and for $\gamma$ any simple closed geodesic $[\gamma] / \ell(\gamma)$ is a boundary point of the unit ball of the stable norm. Denote $L_{\ell}$ the leaf of $\mathcal{T}_{1,1}^{\varepsilon}$ for which $\ell(\gamma)=\ell$.

Lemma 9 Let $\alpha, \beta$ be a pair of distinct geodesics that meet a simple geodesic $\gamma$ in the same number of points. Then for any $\ell>0$, there exists a torus $T \in L_{\ell}$ such that $\ell_{T}(\alpha)=\ell_{T}(\beta)$.

Proof The level sets of the function $f_{\alpha \beta}$ (defined in Lemma 10) are just the FenchelNielsen twist orbits for $\gamma$ and thus are connected. We think of $Z$ as being a zero of the function

$$
M \mapsto \ell_{M}(\alpha)-\ell_{M}(\beta)
$$

where $M$ varies in $L_{\ell}$.

To prove the existence of a solution $Z$ we shall apply the intermediate value theorem on $L_{\ell}$ to our function $f_{\alpha \beta}$. Let $D_{\gamma}$ be a positive Dehn twist along $\gamma$. The Dehn twist acts on both the homotopy class of simple curves and on Teichmiller space, sending a surface $M$ to $D_{\gamma} . M$. For our function this means that

$$
f_{\alpha \beta}\left(D_{\gamma} M\right)=\ell_{M}\left(D_{\gamma}^{-1}(\alpha)\right)-\ell_{M}\left(D_{\gamma}^{-1}(\beta)\right) .
$$

We show that our function changes sign as $M$ varies over a twist orbit by considering the corresponding problem for lengths of curves on a fixed surface $M$; that is, we show that, as $k$ varies over $\mathbb{Z}$, there is a change of sign of

$$
\ell_{M}\left(D_{\gamma}^{-k}(\alpha)\right)-\ell_{M}\left(D_{\gamma}^{-k}(\beta)\right) \text {. }
$$


It is convenient to suppose that $M$ is "rectangular", that is it admits two noncommuting reflections. As before, we choose $\gamma^{\prime}$ so that $\left([\gamma],\left[\gamma^{\prime}\right]\right)$ is a canonical basis of integer homology $H_{1}(T, \mathbb{Z})$ and identify $H_{1}(T, \mathbb{R})$ with $\mathbb{R}^{2}$ with coordinates $(x, y)$ in the obvious way, ie, by sending $[\gamma],\left[\gamma^{\prime}\right]$ to the usual orthonormal basis. The two reflections of $M$ induce automorphisms of $\mathbb{R}^{2}$, reflections in respectively the $x$ and $y$ axes, and $\bar{B}_{1}$, the closed unit ball of the stable norm is invariant under these. Using this invariance one sees that, as we vary over $\bar{B}_{1}$, the maximum $y$ value is attained at $\left[\gamma^{\prime}\right] / \ell_{\gamma^{\prime}}$. Moreover we make the following observation:

Observation If $[\delta],\left[\delta^{\prime}\right]$ on the boundary of $\bar{B}_{1}$ are in the positive quadrant of $\mathbb{R}^{2}$ then the slope of the line joining these points is negative.

Suppose that $\alpha, \beta$ each meet $\gamma$ in exactly $n>0$ points. Then there exist integers $a, b$ such that

and

$$
\begin{aligned}
& {[\alpha]=a[\gamma]+n\left[\gamma^{\prime}\right]} \\
& {[\beta]=b[\gamma]+n\left[\gamma^{\prime}\right] .}
\end{aligned}
$$

After swapping $\alpha, \beta$ we may suppose that $b>a$.

The Dehn twist along $\gamma$ induces an automorphism of $H_{1}(T, \mathbb{Z})$, which extends to $H_{1}(T, \mathbb{R})$, namely

$$
D_{\gamma_{*}}: x[\gamma]+y\left[\gamma^{\prime}\right] \mapsto(x+y)[\gamma]+y\left[\gamma^{\prime}\right] .
$$

To simplify notation for $k \in \mathbb{N}$ we set

$$
\alpha_{k}:=D_{\gamma_{*}}^{k}([\alpha]), \beta_{k}:=D_{\beta_{*}}^{k}([\beta]) .
$$

The corresponding normalized points

$$
\frac{\left[\alpha_{k}\right]}{\ell\left(\alpha_{k}\right)}, \frac{\left[\beta_{k}\right]}{\ell\left(\beta_{k}\right)}
$$

are in the boundary of the unit ball of stable norm [21]. There exists $K \in \mathbb{Z}$ such that for all $k>K$, both $\left[\alpha_{k}\right],\left[\beta_{k}\right]$ are in the positive quadrant of $\mathbb{R}^{2}$. The slope of the line segment joining $\alpha_{k} / \ell_{\alpha_{k}}$ to $\beta_{k} / \ell_{\beta_{k}}$ is

$$
\frac{\ell\left(\alpha_{k}\right) \times b-\ell\left(\beta_{k}\right) \times a}{\ell\left(\alpha_{k}\right)-\ell\left(\beta_{k}\right)},
$$

which is positive if $\ell\left(\beta_{k}\right) \leq \ell\left(\alpha_{k}\right)$ and $b>a$. This contradicts our observation above and so finishes the proof of the lemma. 


\subsection{Uniqueness}

Lemma 10 Consider a leaf $L_{\ell}$ of $\mathcal{T}_{1,1}^{\varepsilon}$ for some fixed $\ell$. Denote by $M_{t}$ the surface of $L_{\ell}$ where the twist parameter along $\gamma$ takes the value $t$. If $a>b$ then the function $f_{\alpha \beta}(t)=\ell_{M_{t}}(\alpha)-\ell_{M_{t}}(\beta)$ is a strictly increasing function.

Remark 9.1 Although $\alpha$ and $\beta$ are oriented curves, this plays no role in the function $f_{\alpha \beta}$.

Proof To prove the lemma, we shall show that the derivative of this function is strictly positive. Clearly, it suffices to show that this holds whenever $a=b+1$, the general case follows by induction.

We have $\iota_{a}(\alpha, \gamma)=\iota_{a}(\beta, \gamma)=n$ and $\iota_{a}\left(\alpha, \gamma^{\prime}\right)-1=\iota_{a}\left(\beta, \gamma^{\prime}\right)=b$. Recall that a simple closed geodesics of a one-holed torus intersect passes through two of the three Weierstrass points of the torus. It follows that any given any pair of simple closed geodesics $\alpha$ and $\beta$ there is a Weierstrass point $p \in \alpha \cap \beta$. Consider the oriented path starting from $p$, following $\beta$ until $\beta$ intersects $\gamma$, then following $\gamma$ until the next intersection point with $\beta$ and so forth. The path closes up after $n$ such steps, when, after having crossed $\gamma n$ times. By construction this is a closed oriented piecewise smooth curve, say $\tilde{\alpha}$, with self intersections occuring only at the points of $\alpha \cap \beta$. Since none of the self intersections of $\widetilde{\alpha}$ are transverse it is homotopic to a simple closed (smooth) curve. We now calculate the algebraic intersection number $\iota(\widetilde{\alpha}, \cdot)$. By construction, $\tilde{\alpha}$ is an Eulerian path for the directed graph with vertices at $\alpha \cap \beta$ and edges the set of all the oriented arcs of both $\gamma$ and $\beta$ joining these points. Thus

$$
\iota_{a}(\tilde{\alpha}, \cdot)=\iota_{a}(\gamma, \cdot)+\iota_{a}(\beta, \cdot) .
$$

It follows that $\iota_{a}(\tilde{\alpha}, \gamma)=\iota_{a}(\gamma, \gamma)+\iota_{a}(\beta, \gamma)=0+n=n$ and $\iota_{a}(\tilde{\alpha}, \delta)=\iota_{a}\left(\gamma, \gamma^{\prime}\right)+$ $\iota_{a}\left(\beta, \gamma^{\prime}\right)=1+b=a$. This proves that $\tilde{\alpha}$ and $\alpha$ determine the same homology class. On a hyperbolic punctured torus there is at most one closed simple geodesic in each homology class so, since $\widetilde{\alpha}$ is homotopic to a simple closed curve, $\widetilde{\alpha}$ and $\alpha$ are homotopic.

Lifting to the universal cover $\mathbb{H}$, we obtain a configuration of curves depicted in Figure 2, where the $\gamma^{(k)}, \beta^{(k)}$ and $p^{(k)}$ correspond to the successive lifts of $\gamma, \beta$ and $p$. So, in the figure, the orientation of $\beta$ is from top to bottom, and the orientation of $\gamma$ from left to right. We obtain a copy of $\alpha$ in $\mathbb{H}$ by joining the points $p^{(1)}$ to $p^{(2)}$ by the unique oriented geodesic arc between them. Denote this oriented geodesic arc by $\alpha^{\prime}$. Note that $p$ may or may not be a point of $\gamma$. Figure 2 portrays the case where $p$ is not a point of $\gamma$. If $p$ was a point of $\gamma$, the lift $\gamma^{(n)}$ would pass through point $p^{(2)}$, and the arc denoted $\beta^{1^{\prime}}$ would not appear. 


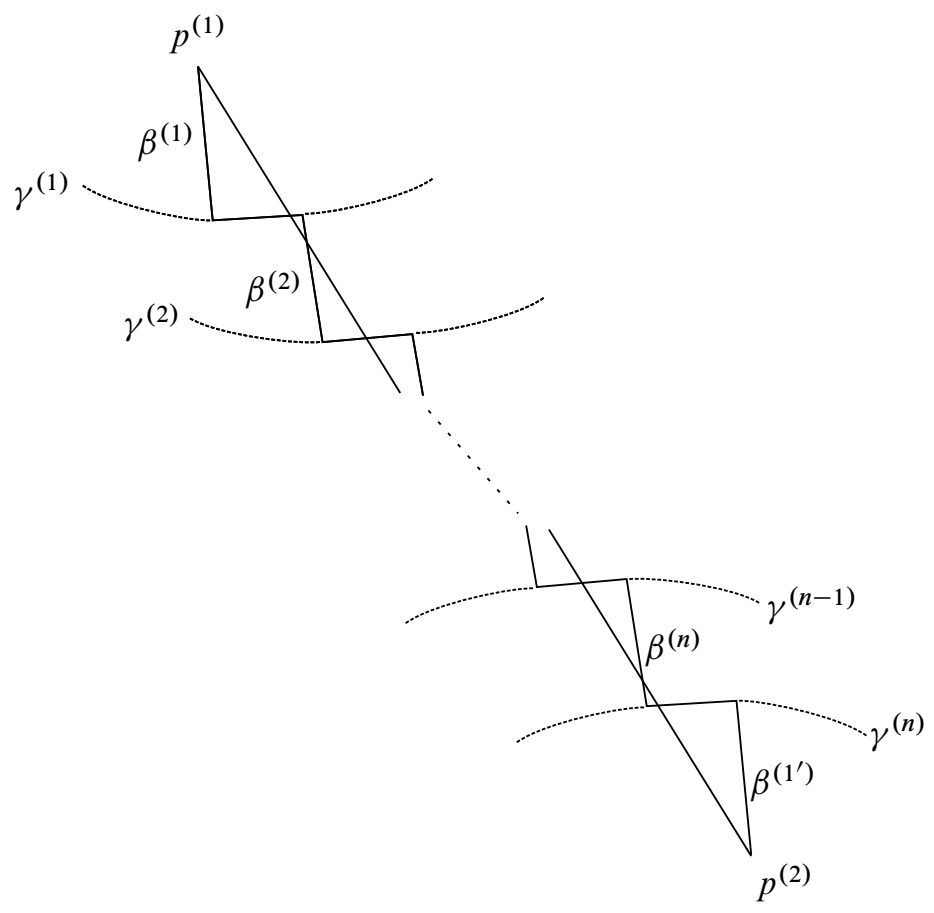

Figure 2: A lift to $\mathbb{H}$

We now put orders on set of $n$ oriented angles of intersection between $\beta$ and $\gamma$, and on the set of $n$ oriented angles of intersection between $\alpha$ and $\gamma$ respectively. Denote by $\left.\theta_{i} \in\right] 0, \pi\left[\right.$ the oriented angle between $\beta^{(i)}$ and $\gamma^{(i)}$, and by $\left.\tilde{\theta}_{i} \in\right] 0, \pi[$ the oriented angle between $\alpha^{(1)}$ and $\gamma^{(i)}$. Now because $\alpha$ is homotopic to $\widetilde{\alpha}$ which is obtained by adding positive arcs along $\gamma$ to $\beta$ we have $\theta_{i}>\tilde{\theta}_{i}$ for all $i \in\{1, \ldots, n\}$. Now by the Kerckhoff-Wolpert formula, the derivative along a positive twist of the function $f_{\alpha \beta}$ is given by $\sum_{k=1}^{n}\left(\cos \tilde{\theta}_{k}-\cos \theta_{k}\right)$ which is always strictly positive as each summand $\cos \tilde{\theta}_{k}-\cos \theta_{k}$ is strictly positive.

Putting the three previous lemmas together, we have now prove Theorem 1.3, which rephrased in the notation introduced above is the following.

Theorem 9.1 Let $\alpha$ and $\beta$ be a pair of distinct simple closed geodesics on a one-holed torus. The set $\mathcal{E}(\alpha, \beta)$ is a simple path joining the points in the Thurston boundary of $\mathcal{T}_{1,1}^{\varepsilon}$ determined by the simple closed geodesics $[\alpha] \pm[\beta]$.

Proof Denote by $L_{\ell}$ the leaf of $\mathcal{T}_{1,1}^{\varepsilon}$ where $\ell(\gamma)=\ell$ and by $L_{\ell}^{\prime}$ the leaf of $\mathcal{T}_{1,1}^{\varepsilon}$ where $\ell\left(\gamma^{\prime}\right)=\ell$. By the previous lemmas, on each leaf $L_{\ell}$ and $L_{\ell}^{\prime}$, there is a unique one-holed torus $T$ where $\ell_{T}(\alpha)=\ell_{T}(\beta)$. As both sets $\left\{L_{\ell}\right\}_{\ell \in] 0, \infty[}$ foliate $\mathcal{T}_{1,1}^{\varepsilon}$, it 
follows that $\mathcal{E}(\alpha, \beta)$ is a simple path between the boundary points of $\mathcal{T}_{1,1}^{\varepsilon} \gamma$, resp. $\gamma^{\prime}$, ie the point where $\ell(\gamma)=0$, respectively the point where $\ell\left(\gamma^{\prime}\right)=0$.

\section{Concluding remarks, problems}

In conclusion we remark that the above situation, for reasons which should be apparent from our treatment, bears a striking similarity to the case of the transcendental numbers. It is relatively easy to establish the existence of aggregate of all transcendentals as a nonempty set, and even to show that in fact most numbers are transcendental. However, to say whether a particular number is transcendental is a very difficult proposition.

Let us end with a list of related problems.

\section{Problems}

(1) It is easy to show that there exists a Euclidean torus, with no symmetries other than the hyperelliptic involution, but which has infinitely many distinct pairs of simple geodesics of the same length; that is it lies on infinitely many different hypersurfaces $\mathcal{E}(.$, .). (It is perhaps surprising that a countable set of planes should have its set of intersections distributed so that they clump together in such a fashion.) Is there a hyperbolic once-punctured torus with this property?

(2) On the other hand is there a number $K>1$ such that if $M$ has $K$ pairs of equal length then $M$, a hyperbolic torus, must have an isometry (other than the hyperelliptic involution)?

(3) We have constructed a dense set of counterexamples to the naive geometric generalization of the Markoff conjecture. That is, for a dense set a of $t \geq 3$, there are matrices, in $S L(2, \mathbb{R}), A_{t}, B_{t}, A_{t} B_{t}=C_{t} \in S L(2, \mathbb{R})$, each with trace $t$, such that the quotient of $\mathbb{H}$ by the Fuchsian group they generate is a torus with a hole with maximal symmetry group but the multiplicity of the simple spectrum is at least 12 . Is it possible to find such a $t \in \mathbb{Z}$ ?

(4) As mentioned in the introduction, it is not known if simple multiplicity is always bounded for a hyperbolic surface. This is probably a very difficult problem. To illustrate the difficulty, let us make the following observation. Recent results on both upper and lower polynomial bounds on the growth of simple closed geodesics in function of length (see Mirzakhani [22] and Rivin [24]) imply that on any hyperbolic surface which is not a pair of pants, any $\varepsilon>0$ and any $n$, there exists $n$ distinct simple closed geodesics $\gamma_{1}, \ldots, \gamma_{n}$ such that $\left|\ell\left(\gamma_{k}\right)-\ell\left(\gamma_{l}\right)\right|<\varepsilon$ for all $k, l \in\{1, \ldots, n\}$. To prove this, it suffices to notice that if this were not true, then there would be a linear growth bound. 


\section{Appendix A Proofs of Theorems 2.1 and 2.2}

We begin by treating the case of one-holed tori. In this case, the map from Teichmüller space to the set of lengths of interior simple closed geodesics is projectively injective.

Lemma 11 There are four interior simple closed curves $\alpha, \beta, \gamma$, and $\delta$ of a oneholed torus such that the map $\varphi: M \mapsto\left(\ell_{M}(\alpha), \ell_{M}(\beta), \ell_{M}(\gamma), \ell_{M}(\delta)\right)$ is projectively injective.

Proof Let $M$ be a one-holed torus and let $\alpha, \beta, \gamma$, and $\delta$ be the simple closed curves as in Figure 3.
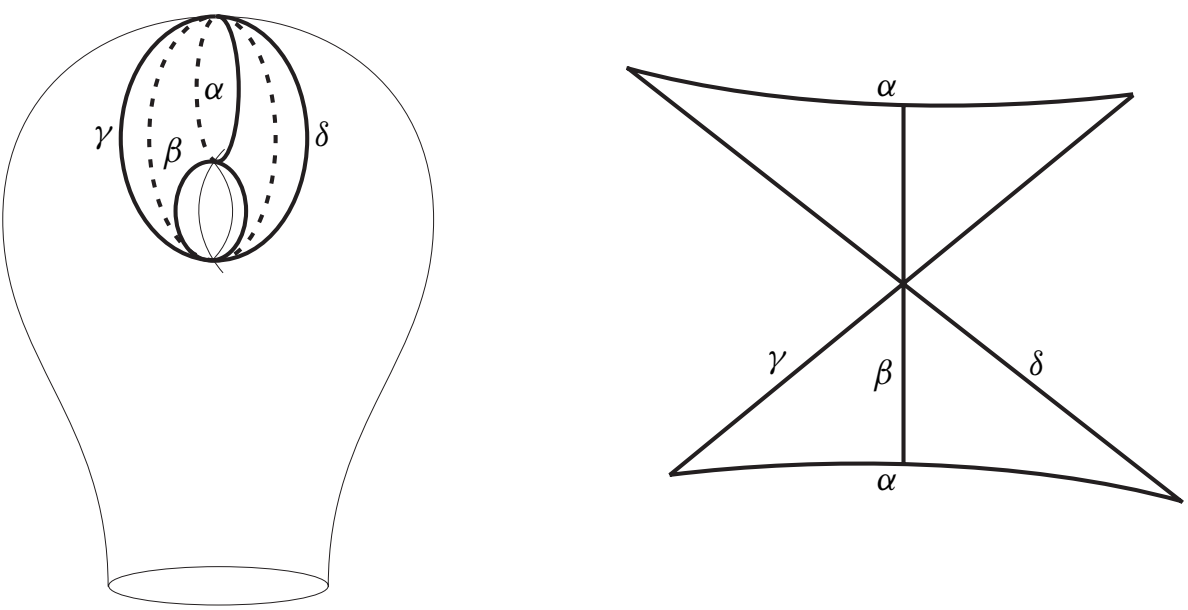

Figure 3: The one-holed torus with four interior geodesics and the four curves seen in the universal cover

The remarkable fact about the geodesic representatives of simple closed curves on a one-holed torus is that they pass through exactly two of the three Weierstrass points of the torus in diametrically opposite points. In the case of the curves $\alpha, \beta, \gamma$, and $\delta$, their intersection points are all Weierstrass points. Therefore they can be seen in the universal cover as in Figure 3. It is well known that the lengths of $\alpha, \beta$, and $\gamma$ determine a unique point in Teichmüller space (see for example Buser and Semmler [6]). The lengths of these three curves up to a multiplicative constant do not determine a unique point in Teichmüller space however. For this we need the curve $\delta$. What we need to prove is that if we have two one-holed tori $M_{1}$ and $M_{2}$ in Teichmüller space with

$$
\left(\ell_{M_{1}}(\alpha), \ell_{M_{1}}(\beta), \ell_{M_{1}}(\gamma), \ell_{M_{1}}(\delta)\right)=\lambda\left(\ell_{M_{2}}(\alpha), \ell_{M_{2}}(\beta), \ell_{M_{2}}(\gamma), \ell_{M_{2}}(\delta)\right)
$$


for some $\lambda \in \mathbb{R}$, then $\lambda=1$ and then, by what precedes, $M_{1}=M_{2}$. Figure 3 shows four hyperbolic triangles. Consider the two bottom ones. The side lengths of the bottom left triangle are $\ell(\alpha) / 2, \ell(\beta) / 2$, and $\ell(\gamma) / 2$. The side lengths of the bottom right triangle are $\ell(\alpha) / 2, \ell(\beta) / 2$, and $\ell(\delta) / 2$. The bottom intersection point between $\alpha$ and $\beta$ forms two angles depending on the surface $M$, say $\theta_{1}(M)$ and $\theta_{2}(M)$ such that $\theta_{1}+\theta_{2}=\pi$. Suppose without loss of generality that $\lambda \geq 1$. Now if for $M_{1}$ the triangle lengths are equal to $a, b, c$ and $d$, the triangle lengths for $M_{2}$ are $\lambda a, \lambda b, \lambda c$ and $\lambda d$. This implies that $\theta_{1}\left(M_{1}\right) \leq \theta_{1}\left(M_{2}\right)$ as well as $\theta_{2}\left(M_{1}\right) \leq \theta_{2}\left(M_{2}\right)$, equality occurring only if $\lambda=1$. As $\theta_{1}+\theta_{2}$ is always equal to $\pi$, this concludes the proof.

In order to prove the more general theorems, we shall need some lemmas from elementary real function theory concerning sums of logarithmic and exponential functions. The functions we shall study arise naturally as trace polynomials satisfied by traces of simple closed geodesics. (Recall the trace of a simple closed geodesic $\gamma$ is the trace of a matrix in $\operatorname{PSL}(2, \mathbb{R})$ which covers $\gamma$ and is equal to $2 \cosh (\ell(\gamma) / 2)$.)

Lemma 12 Let $F: \mathbb{R}^{n} \rightarrow \mathbb{R}$ be the following function in $n>1$ variables:

$$
F(x)=\log \left(\sum_{k=1}^{n} x_{k}-1\right)\left(\sum_{k=1}^{n} x_{k}-1\right)-\sum_{k=1}^{n} x_{k} \log x_{k} .
$$

Then $F(x) \geq 0$ for all $x$ with $\min _{k=1, \ldots, n} x_{k} \geq 1$.

Proof We have $F(1, \ldots, 1)=0$ and $\partial F(x) / \partial x_{l}=\log \left(\sum_{k=1}^{n} x_{k}-1\right)-\log x_{l} \geq 0$ for all $l \in\{1, \ldots, n\}$ which proves the result.

Lemma 13 Let $a_{1}, a_{2}, b_{1}, \ldots, b_{n}$ be positive numbers satisfying $a_{1}>b_{k}$ for all $k \in\{1, \ldots, n\}$, and let $f$ be the following real function defined for $t \in \mathbb{R}^{+}$:

$$
f(t)=a_{1}^{t}+a_{2}^{t}-\sum_{k=1}^{n} b_{k}^{t}
$$

Then $f$ has at most one strictly positive zero.

Proof We can suppose that $a_{2}=1$ as multiplying $f$ by $a_{2}^{-t}$ does not change the sign of $f$, nor does it change the order of the positive numbers. 
Let us suppose that $f(t) \geq 0$ for some $t>0$. We shall show that this implies $f^{\prime}(t) \geq 0$. By calculation

$$
\begin{gathered}
f^{\prime}(t)=a_{1}^{t} \log a_{1}-\sum_{k=1} n b_{k}^{t} \log b_{k} \\
t f^{\prime}(t)=A_{1} \log A_{1}-\sum_{k=1}^{n} B_{k} \log B_{k}
\end{gathered}
$$

and thus

with $A_{1}=a_{1}^{t}$ and $B_{k}=b_{k}^{t}$. Because $f(t) \geq 0$, we have $A_{1} \geq \sum_{k=1}^{n} B_{k}-1$, and it follows that

$$
t f^{\prime}(t) \geq \log \left(\sum_{k=1}^{n} B_{k}-1\right)\left(\sum_{k=1}^{n} B_{k}-1\right)-\sum_{k=1}^{n} B_{k} \log B_{k} .
$$

If $B_{k} \geq 1$ for $k \in\{1, \ldots, n\}$, then by Lemma $12, t f^{\prime}(t) \geq 0$. If not, then notice that

$$
t f^{\prime}(t) \geq \log \left(\sum_{k=1}^{\tilde{n}} B_{k}-1\right)\left(\sum_{k=1}^{\tilde{n}} B_{k}-1\right)-\sum_{k=1}^{\tilde{n}} B_{k} \log B_{k}
$$

with $\tilde{n}<n$ and all $B_{k} \geq 1$ for $k \leq \tilde{n}$ is positive if $\tilde{n} \geq 2$. Now if $\tilde{n} \leq 1$ then $a_{1}>b_{1}$ and $a_{2}=1>b_{2}$ and the result is trivial.

Corollary A.1 Let $a_{1}, a_{2}, b_{1}, \ldots, b_{n}$ be positive numbers satisfying $a_{1}>b_{k}$ for all $k \in\{1, \ldots, n\}$, and let $f$ be the following real function defined for $t \in \mathbb{R}^{+}$:

$$
f(x)=\cosh \left(a_{1} x\right)+\cosh \left(a_{2} x\right)-\sum_{k=1}^{n} \cosh \left(b_{k} x\right) .
$$

Then $f$ has at most one strictly positive zero.

Proof Let us suppose that $a_{1} \geq a_{2}$. Let us remark that $\lim _{x \rightarrow+\infty} f(x)=+\infty$. By calculation

$$
f^{(2 p)}(x)=a_{1}^{2 p} \cosh \left(a_{1} x\right)+a_{2}^{2 p} \cosh \left(a_{2} x\right)-\sum_{k=1}^{n} b_{k}^{2 p} \cosh \left(b_{k} x\right)
$$

and $f^{(2 p+1)}(x)=a_{1}^{2 p+1} \sinh \left(a_{1} x\right)+a_{2}^{2 p+1} \sinh \left(a_{2} x\right)-\sum_{k=1}^{n} b_{k}^{2 p+1} \sinh \left(b_{k} x\right)$.

Because $a_{1}>b_{k}$ for all $k \in\{1, \ldots, n\}$, it follows that there exists a $p$ such that $a_{1}^{2 p}>$ $\sum_{k=1}^{n} b_{k}^{2 p}$ and it follows that for this $p$, we have $f^{(2 p)}(x)>0$ for all $x \geq 0$. We shall proceed backwards to $f$. Notice that for any $q \in \mathbb{N}, f^{(2 q)}(0)=a_{1}^{2 q}+a_{2}^{2 q}-\sum_{k=1}^{n} b_{k}^{2 q}$ 
and that $f^{(2 q+1)}(0)=0$. It follows that $f^{(2 p-1)}(x)>0$ for all $t>0$. For all $k$ less than $2 p-1, f^{(k)}(t)>0$ for all $t>0$, until there is a $p_{1}$ such that $f^{\left(2 p_{1}\right)}(0)<0$. (If such a $p_{1}$ does not exist then $f$ is a strictly increasing function and the result is obvious.) Notice that the existence of $p_{1}$ implies that the function defined as $g(t):=f(t)=a_{1}^{t}+a_{2}^{t}-\sum_{k=1}^{n} b_{k}^{t}$ has a positive zero between $2 p_{1}$ and $2 p-1$. By Lemma 13 , this is the unique strictly positive zero of the function $g$. The unicity of this zero implies that for $k=1, \ldots, p_{1}-1$ we have $f^{(2 k)}(0)<0$. Also, $f^{(2 k+1)}(x)$ takes value 0 for $x=0$, is strictly decreasing for a while, and then is strictly increasing and goes to infinity. In particular, $f^{\prime}(x)$ behaves this way. As $f(0)<0$, this completes the proof.

We are now set to prove Theorem 2.1. Let us recall its statement.

Theorem 2.1 There is a fixed finite set of simple closed geodesics $\gamma_{1}, \ldots, \gamma_{n}$ such that the map $\varphi: M \mapsto\left(\ell_{M}\left(\gamma_{1}\right), \ldots, \ell_{M}\left(\gamma_{n}\right)\right)$ is projectively injective.

Proof The first step is to show that the lengths of a finite set of simple closed geodesics determine a point in Teichmüller space. Recall that a point in Teichmüller space is determined by the lengths of the geodesics of a pants decomposition and twist parameters along the interior geodesics of the pants decomposition. In turn, the twist parameter around a geodesic is determined by the lengths of two simple closed geodesics that intersect the pants geodesic minimally, that mutually intersect minimally and do not intersect any other geodesic of the pants decomposition. (For a proof of how these lengths determine the twist parameter, see for example Buser [5]). Thus the geodesics of a pants decomposition can be completed into a finite set of simple closed geodesics, say $\left\{\gamma_{1}, \ldots, \gamma_{m}\right\}$, whose lengths determines a unique point in Teichmüller space.

We shall now show that we can add an extra geodesic to one of the finite sets described above such that the map to the lengths of the extended set is projectively injective. There will be two cases to consider: either the surface is of signature $(g, n)$ with $g>0$, or not. In the first case, the surface has an embedded one-holed torus, whose boundary curve we ensure is in the simple closed geodesics of our pants decomposition used to create the above set. By Lemma 11, it suffices to add an simple closed geodesic $\gamma_{m+1}$ which is interior of our torus, and the result follows.

Now if $g=0$, for any pants decomposition, the surface contains an embedded four holed sphere whose boundary geodesics are among the pants decomposition geodesics of the surface. Denote by $\alpha, \beta, \gamma, \delta$ these boundary geodesics, and by $\zeta, \xi$ and $v$ the interior geodesics of the finite set determined above. Consider an auxiliary geodesic $\bar{\xi}$ which is only simple closed geodesic with two intersection points with both $\zeta$ and $v$ 

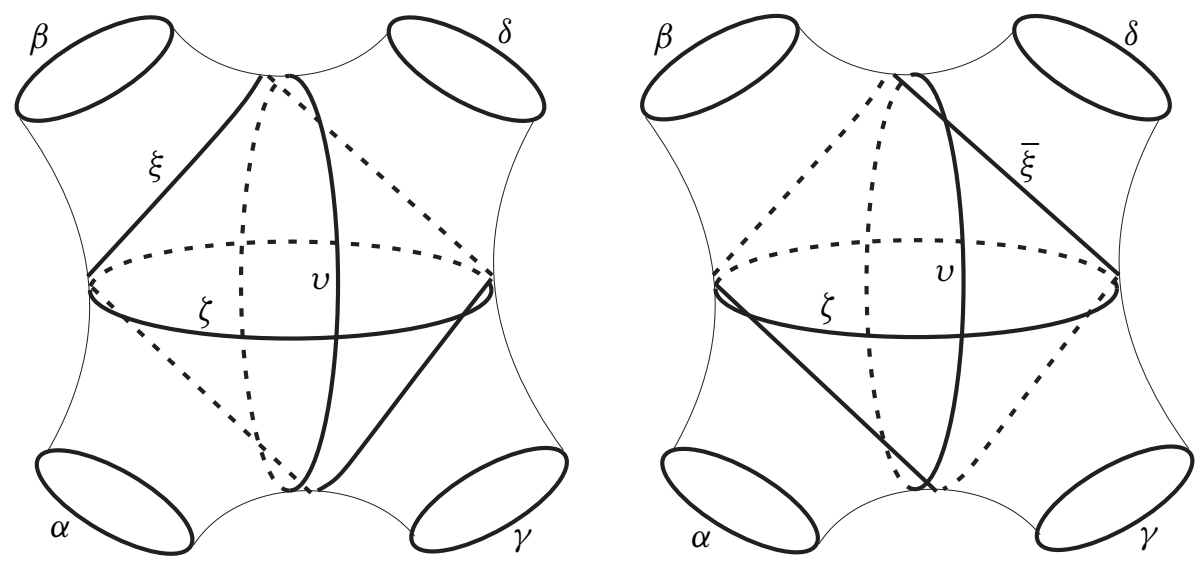

Figure 4: The geodesics $\zeta, v, \xi$ and $\bar{\xi}$ on the four holed sphere

distinct from $\xi$. It necessarily has four intersection points with $\xi$, and is portrayed in Figure 4.

In [13], it is shown that the geodesics described above satisfy a certain number of trace equalities. As we require information on the lengths of the corresponding geodesics, we have translated these equations accordingly. One of them, that is used to describe a fundamental domain for action of the mapping class group of the four-holed sphere, is the following:

$$
\begin{aligned}
\cosh \frac{\ell(\xi)}{2}+\cosh \frac{\ell(\bar{\xi})}{2}+2 & \left(\cosh \frac{\ell(\alpha)}{2} \cosh \frac{\ell(\delta)}{2}+\cosh \frac{\ell(\beta)}{2} \cosh \frac{\ell(\gamma)}{2}\right) \\
= & 2 \cosh \frac{\ell(v)}{2} \cosh \frac{\ell(\zeta)}{2} .
\end{aligned}
$$

By hyperbolic trigonometry this becomes

$$
\begin{aligned}
\cosh \frac{\ell(\xi)}{2}+\cosh \frac{\ell(\bar{\xi})}{2}+\cosh \frac{\ell(\alpha)+\ell(\delta)}{2}+ & \cosh \frac{\ell(\alpha)-\ell(\delta)}{2} \\
+\cosh \frac{\ell(\beta)+\ell(\gamma)}{2}+ & \cosh \frac{\ell(\beta)-\ell(\gamma)}{2} \\
& =\cosh \frac{\ell(v)+\ell(\zeta)}{2}+\cosh \frac{\ell(v)-\ell(\zeta)}{2} .
\end{aligned}
$$

Notice that for geometric reasons, $\cosh ((\ell(v)+\ell(\zeta)) / 2)$ is greater than all of the other summands. Suppose now that the map on the lengths was not projectively injective. That would imply that there exists at least two surfaces with their lengths satisfying the above equations. In other words, the above equations are satisfied by both $\alpha, \beta, \gamma, \delta, \zeta, v, \xi, \bar{\xi}$ and $t_{0}(\alpha, \beta, \gamma, \delta, \zeta, v, \xi, \bar{\xi})$ for some $t_{0}$. Now consider the function 


$$
\begin{aligned}
f(t):=\sum_{k=1}^{2} \cosh \left(a_{k} t\right)-\sum_{k=1}^{6} \cosh \left(b_{k} t\right) \text { with } \\
a_{1}:=\frac{\ell(v)+\ell(\zeta)}{2}, \\
a_{2}:=\frac{\ell(v)-\ell(\zeta)}{2}, \\
b_{1}:=\frac{\ell(\xi)}{2}, \\
b_{2}:=\frac{\ell(\bar{\xi})}{2}, \\
b_{3}:=\frac{\ell(\alpha)+\ell(\delta)}{2}, \\
b_{4}:=\frac{\ell(\alpha)-\ell(\delta)}{2}, \\
b_{5}:=\frac{\ell(\beta)+\ell(\gamma)}{2}, \\
b_{6}:=\frac{\ell(\beta)-\ell(\gamma)}{2} .
\end{aligned}
$$

Notice that the constants $a_{1}, a_{2}, b_{1}, \ldots, b_{6}$ satisfy the conditions of Corollary A.1. The equation $f(t)=0$ has a positive solution given by $t=1$, and by Corollary A.1, this solution is unique (given by $t_{0}=1$ ) which completes the proof.

Remark A.1 The number of curves (denoted $n$ above) in the projectively injective map which we obtain is in no way optimal. In the case of surfaces of genus $g$ with $m$ cusps, Hamenstädt has shown that the optimal number is $6 g-5+2 m$ [17].

Consider a four-holed sphere. For a given choice of $\zeta, v$ and $\xi$, the simple closed geodesic $\bar{\xi}$ is uniquely determined. Similarly, consider the auxiliary curves $\bar{v}$ and $\bar{\zeta}$ as the unique simple closed geodesics (distinct from respectively $v$ and $\zeta$ ) such that $\bar{v}$ intersects both $\xi$ and $\zeta$ twice, respectively such that $\bar{\zeta}$ intersects both $\xi$ and $v$ twice. As before, notice that $v$ and $\bar{v}$ intersect four times, respectively that $\zeta$ and $\bar{\zeta}$ intersect four times. We now have a set of six interior simple closed geodesics $\{\xi, \bar{\xi}, v, \bar{v}, \zeta, \bar{\zeta}\}$ whose lengths are shown in the following lemma to determine a finite number of points in Teichmüller space.

Lemma 14 Let $\mathcal{T}$ be the Teichmüller space of the four-holed sphere, and let $M \in \mathcal{T}$. Then there are finitely many $M_{k} \in \mathcal{T}$ such that $\ell_{M}(\gamma)=\ell_{M_{k}}(\gamma)$ for all $\gamma \in$ $\{\xi, \bar{\xi}, v, \bar{v}, \zeta, \bar{\zeta}\}$. 
Proof The idea is to show that the lengths of the six curves $\{\xi, \bar{\xi}, v, \bar{v}, \zeta, \bar{\zeta}\}$ determine a finite number of possibilities for the lengths of the four boundary curves $\alpha, \beta, \gamma$ and $\delta$. In the proof of the previous theorem, we have seen that the lengths of the geodesic representatives of $\xi, \bar{\xi}, v, \zeta$ satisfy an interesting trace equation (Equation $(\mathrm{A}-1))$ involving the boundary geodesics. The sets $\xi, v, \bar{v}, \zeta$ and $\xi, v, \zeta, \bar{\zeta}$ satisfy similar equations (Equations $(\mathrm{A}-5)$ and (A-6) below). All these equations, along with an additional trace equality which we shall detail later (Equation $(A-3)$ ), have been proved in [13] and are the basis of this proof. We shall use the following abbreviations for these equations $a=\cosh (\ell(\alpha) / 2$ ) etc. (In other words, the half-trace of a curve denoted by a Greek letter is denoted by the lowercase corresponding roman letter.) The additional trace equation mentioned earlier is the following:

$$
\begin{aligned}
a^{2}+b^{2}+c^{2} & +d^{2}+x^{2}+y^{2}+z^{2}+4 a b c d-1 \\
& -2 x y z+2 x(a d+b c)+2 y(a b+c d)+2 z(a c+b d)=0 .
\end{aligned}
$$

The full list of equalities that we shall use is:

$$
\begin{aligned}
& a d+b c=y z-\frac{x+\bar{x}}{2}, \\
& a c+b d=x y-\frac{z+\bar{z}}{2}, \\
& a b+c d=x z-\frac{y+\bar{y}}{2},
\end{aligned}
$$

$$
a^{2}+b^{2}+c^{2}+d^{2}+4 a b c d=1-4 x y z+x \bar{x}+y \bar{y}+z \bar{z} .
$$

(The last equation is obtained from an obvious manipulation of $(A-3)$ and $(A-4),(A-5)$, (A-6).) Notice that the right-hand side of each equation is determined by the lengths of $\{\xi, \bar{\xi}, v, \bar{v}, \zeta, \bar{\zeta}\}$. Denote by $f_{1}, \ldots, f_{4}$ the right hand side of each equation, which we shall view as given constants. It now suffices to show that for given $f_{1}, \ldots, f_{4} \in \mathbb{R}^{+*}$, there are a finite number of $(a, b, c, d) \in\left(\mathbb{R}^{+*}\right)^{4}$ solution to the system of equations. There are five distinct situations to consider.

The first situation is when $a=b=c=d$, which implies $f_{1}=f_{2}=f_{3}$ and $a=b=$ $c=d=\sqrt{f_{1} / 2}$.

If $a=b=c$, then

$$
\begin{aligned}
a^{2}+a d & =f_{1}=f_{2}=f_{3}, \\
3 a^{2}+d^{2}+4 a^{3} d & =f_{4} .
\end{aligned}
$$

From these equations, we can deduce a single polynomial equation in $a$ with a finite number of solutions. Working backwards, one obtains a finite number of solutions $(a=b=c, d)$ to Equation $(\mathrm{A}-4)$. 
Now if $a=b$ and $c=d$ then

$$
\begin{aligned}
a+c & =\sqrt{f_{1}+f_{2}}, \\
a c & =\frac{f_{2}}{2},
\end{aligned}
$$

which trivially implies a finite (but not unique) set of solutions of type $(a, a, c, c)$.

The two remaining cases (when $a=b, a \neq c, d, c \neq d$, and when all four variables $a, b, c, d$ are distinct) are similar in nature. We shall give the full solution to the latter case which is the most complicated and leave the remaining case to the dedicated reader. Note that $a, b, c, d$ distinct implies that the constants $f_{1}, f_{2}$ and $f_{3}$ are also distinct. By manipulating the system of equations one obtains

$$
\begin{aligned}
& a=\frac{f_{2} c-f_{1} d}{c^{2}-d^{2}}, \\
& b=\frac{f_{1} c-f_{2} d}{c^{2}-d^{2}},
\end{aligned}
$$

$(\mathrm{A}-10) \quad\left(c d-f_{3}\right)\left(c^{2}-d^{2}\right)^{2}+\left(f_{1} c-f_{2} d\right)\left(f_{2} c-f_{1} d\right)=0$,

$$
\begin{aligned}
\left(f_{2} c-f_{1} d\right)^{2} & +\left(f_{1} c-f_{2} d\right)^{2}+\left(d^{2}+c^{2}\right)\left(c^{2}-d^{2}\right)^{2} \\
& +4\left(f_{2} c-f_{1} d\right)\left(f_{1} c-f_{2} d\right) c d-f_{4}\left(c^{2}-d^{2}\right)^{2}=0 .
\end{aligned}
$$

Equations (A-10) and (A-11) determine two real planar curves. There are a finite number of pairs $(c, d)$ solution to both equations if and only if their underlying polynomials are coprime in $\mathbb{R}[c, d]$. In order to determine whether or not they polynomials are coprime, we shall consider the polynomials as polynomials in variable $c$, resp. in variable $d$. Note that if the two polynomials, say $P$ and $Q$, are not coprime in $\mathbb{R}[c, d]$, then they are not coprime in either $\mathbb{R}[c]$ or $\mathbb{R}[d]$. We shall then calculate the resultant of the two polynomials in $c$, resp. in $d$, which gives a polynomial $R_{c}(d)$ in variable $d$, resp. $R_{d}(c)$ in variable $c$. We shall see that neither of the polynomials $R_{c}(d)$ and $R_{d}(c)$ are identically zero. This implies that $P$ and $Q$ are coprime in both $\mathbb{R}[c]$ and $\mathbb{R}[d]$, which in turn implies that they are coprime in $\mathbb{R}[c, d]$. We have

$$
\begin{gathered}
P=\left(c d-f_{3}\right)\left(c^{2}-d^{2}\right)^{2}+\left(f_{1} c-f_{2} d\right)\left(f_{2} c-f_{1} d\right), \\
Q=\left(f_{2} c-f_{1} d\right)^{2}+\left(f_{1} c-f_{2} d\right)^{2}+\left(d^{2}+c^{2}\right)\left(c^{2}-d^{2}\right)^{2} \\
\quad+4\left(f_{2} c-f_{1} d\right)\left(f_{1} c-f_{2} d\right) c d-f_{4}\left(c^{2}-d^{2}\right)^{2} .
\end{gathered}
$$

The resultants are calculated by calculating the determinants of the associated Sylvester matrixes of the two polynomials. In our case, by computation, the resultant $R_{c}(d)=$ $\sum_{k=1}^{28} \alpha_{k} d^{k}$ is a degree 28 polynomial with many terms, but luckily, the leading 
coefficient is simple and equal to

$$
\alpha_{28}=256\left(f_{1}-f_{2}\right)^{4}\left(f_{1}+f_{2}\right)^{4} .
$$

Similarly (and by symmetry), $R_{d}(c)=\sum_{k=1}^{28} \beta_{k} d^{k}$, and

$$
\beta_{28}=256\left(f_{1}-f_{2}\right)^{4}\left(f_{1}+f_{2}\right)^{4} .
$$

As $f_{1} \neq f_{2}$ and both $f_{1}$ and $f_{2}$ are strictly positive, this proves the result. The remaining case can be solved using the same method.

Remark A.2 One might hope for unicity in the previous lemma (as in the case of the one-holed torus, Lemma 11), ie, that the lengths of our well chosen six interior simple closed curves determine uniquely the lengths of the boundary geodesics and thus a unique point in Teichmüller space. A first remark is that, by using the trace equalities used above, the length of the six curves $\{\xi, \bar{\xi}, v, \bar{v}, \zeta, \bar{\zeta}\}$ determine the lengths of all interior simple closed geodesics of a given surface (see Gauglhofer and Semmler [13] for a full proof). In spite of this, it is easy to construct examples of pairs of marked four holed spheres where the lengths of $\{\xi, \bar{\xi}, v, \bar{v}, \zeta, \bar{\zeta}\}$ are equal but the surfaces represent different points in $\mathcal{T}$. To do this consider a surface $M_{1}$ with marked boundary lengths $\ell_{M_{1}}(\alpha)=\ell_{M_{1}}(\beta)=\ell_{1}$, and $\ell_{M_{1}}(\gamma)=\ell_{M_{1}}(\delta)=\ell_{2}$. Further consider that the marked surface $M_{1}$ has twist parameter equal to 0 along $v$. Now consider a surface $M_{2}$ with boundary lengths $\ell_{M_{2}}(\alpha)=\ell_{M_{2}}(\beta)=\ell_{2}$ and $\ell_{M_{2}}(\gamma)=\ell_{M_{2}}(\delta)=\ell_{1}$, satisfying $\ell_{M_{2}}(v)=\ell_{M_{1}}(v)$, also with twist parameter 0 along $v$. It is not too difficult to see that the lengths of $\bar{v}, \xi, \bar{\xi}, \zeta$ and $\bar{\zeta}$ are the same for both $M_{1}$ and $M_{2}$, but yet $M_{1}$ and $M_{2}$ are clearly distinct points in $\mathcal{T}$. These examples, however, are examples of the same surface up to isometry. What is more surprising is that one can find examples of surfaces that are not isometric, but have the same interior length spectrum.

Example A.1 Consider a four holed sphere $\widetilde{M}_{1}$ whose marked boundary lengths are $\ell_{\widetilde{M}_{1}}(\alpha)=\ell_{\widetilde{M}_{1}}(\beta)=\ell_{\widetilde{M}_{1}}(\gamma)=2 \operatorname{arccosh} 2$, and $\ell_{\widetilde{M}_{1}}(\delta)=2 \operatorname{arccosh} 3$. Now set our marked interior geodesic lengths to

$$
\ell \widetilde{M}_{1}(v)=\ell_{\widetilde{M}_{1}}(\xi)=\ell_{\widetilde{M}_{1}}(\zeta)=q,
$$

where

$$
q=2 \operatorname{arccosh}\left(1+(293-92 \sqrt{2})^{\frac{1}{3}}+(293+92 \sqrt{2})^{\frac{1}{3}}\right) .
$$

This surface has the same interior length spectrum as the surface $\widetilde{M}_{2}$ with

$$
\ell_{\widetilde{M}_{2}}(v)=\ell_{\widetilde{M}_{2}}(\xi)=\ell_{\widetilde{M}_{2}}(\zeta)=q,
$$


but with $\ell_{\widetilde{M}_{2}}(\alpha)=\ell \widetilde{M}_{2}(\beta)=\ell \widetilde{M}_{2}(\gamma)=r$, and $\ell_{\widetilde{M}_{2}}(\delta)=s$ where

and

$$
\begin{aligned}
& r=2 \operatorname{arccosh} \sqrt{\frac{7}{2}-\sqrt{6}}, \\
& s=2 \operatorname{arccosh} \sqrt{\frac{79}{2}+15 \sqrt{6}} .
\end{aligned}
$$

To see that these two surfaces have the interior simple length spectrum, by the above it suffices to check that the left-hand sides of Equations (A-8), (A-9), and (A-10) are the same, and they are by calculation. This example is in no way isolated, and the trick to finding it was to search for two surfaces with distinct boundary lengths, but each with three equal boundary lengths. With this method you get at least a real dimension 1 family of such surfaces.

We are now ready to prove Theorem 2.2.

Theorem 2.2 Let $\mathcal{T}$ be the Teichmüller space of given signature. Then, there is a set $\Gamma:=\left\{\gamma_{1}, \ldots, \gamma_{n}\right\}$ of interior simple closed curves such that for any given point in $M \in \mathcal{T}$, there are a finite number of $M^{\prime} \in \mathcal{T}$ such that $\lambda\left(\ell_{M}\left(\gamma_{1}\right), \ldots, \ell_{M}\left(\gamma_{n}\right)=\right.$ $\left(\ell_{M^{\prime}}\left(\gamma_{1}\right), \ldots, \ell_{M^{\prime}}\left(\gamma_{n}\right)\right)$ for some $\lambda \in \mathbb{R}$.

Proof This is obviously true by Theorem 2.1 for closed surfaces and by Lemma 11 for the one-holed torus. In the remaining cases, consider the set of geodesics $\Gamma^{\prime}:=$ $\left\{\gamma_{1}, \ldots, \gamma_{m}\right\}$ constructed for the proof of Theorem 2.1. Each boundary curve (of the base surface) in the set $\Gamma^{\prime}$ is a boundary curve of a four-holed sphere formed by elements of $\Gamma^{\prime}$. To $\Gamma^{\prime}$, we need to add auxiliary curves to obtain a set $\Gamma^{\prime \prime}$ such that every boundary curve of $\Gamma^{\prime}$ is the boundary curve of a four-holed sphere whose interior curves $\{\xi, \bar{\xi}, v, \bar{v}, \zeta, \bar{\zeta}\}$ are elements of $\Gamma^{\prime \prime}$. Now consider the set $\Gamma^{\prime \prime \prime}$ is obtained by removing the boundary curves from $\Gamma^{\prime \prime}$. By the previous lemma and the initial considerations in the proof of Theorem 2.1, the lengths of the elements of $\Gamma^{\prime \prime \prime}$ determine a finite number of marked surfaces in $\mathcal{T}$.

We now need to show that the equality

$$
\lambda\left(\ell_{M}\left(\gamma_{1}\right), \ldots, \ell_{M}\left(\gamma_{n}\right)\right)=\left(\left(\ell_{M^{\prime}}\left(\gamma_{1}\right), \ldots, \ell_{M^{\prime}}\left(\gamma_{n}\right)\right)\right.
$$

can only be true for a finite number of $\lambda$ for some choice of $\Gamma:=\left\{\gamma_{1}, \ldots, \gamma_{n}\right\}$. If the genus of the underlying surface is not 0 , and as we have mimicked the set of geodesics in Theorem 2.1, by Lemma 11 it follows that $\lambda=1$. Otherwise, let us consider one of the four-holed spheres whose interior curves $\{\xi, \bar{\xi}, v, \bar{v}, \zeta, \bar{\zeta}\}$ are in $\Gamma^{\prime \prime \prime}$. Consider the further set of interior curves obtained by a single right Dehn twist around $\zeta$ of the curves $\xi, \bar{\xi}, v$ and $\zeta$. Notice the curve obtained from $\zeta$ this way is of course itself, 
and the curve obtained by $\xi$ is $\bar{\xi}$. Denote by $\bar{\xi}^{\prime}$ and $v^{\prime}$ the images of $\bar{\xi}$ and $v$. We can now fix $\Gamma:=\Gamma^{\prime \prime \prime} \cup\left\{\bar{\xi}^{\prime}, v^{\prime}\right\}$. Recall that the lengths of $\xi, \bar{\xi}, v$ and $\zeta$ satisfy equality (A-2). As a Dehn twist along $\zeta$ does not change the equality satisfied by the set of curves, this gives a second equality which, with Equation (A-2), gives:

$$
\begin{aligned}
& \cosh \frac{\ell(\xi)}{2}+\cosh \frac{\ell\left(v^{\prime}\right)+\ell(\zeta)}{2}+\cosh \frac{\ell\left(v^{\prime}\right)-\ell(\zeta)}{2} \\
= & \cosh \frac{\ell\left(\bar{\xi}^{\prime}\right)}{2}+\cosh \frac{\ell(v)+\ell(\zeta)}{2}+\cosh \frac{\ell(v)-\ell(\zeta)}{2} .
\end{aligned}
$$

Now suppose $\ell_{M_{1}}(\Gamma)=\lambda \ell_{M_{2}}(\Gamma)$. This implies that $f(1)=0$ and $f(\lambda)=0$ for

$$
f(t):=\sum_{k=1}^{3} \cosh \left(a_{k} t\right)-\sum_{k=1}^{3} \cosh \left(b_{k} t\right),
$$

with

$$
\begin{aligned}
a_{1} & =\frac{\ell_{M}(\xi)}{2}, \\
a_{2} & =\frac{\ell_{M}\left(v^{\prime}\right)+\ell_{M}(\zeta)}{2} \\
a_{3} & =\frac{\ell_{M}\left(v^{\prime}\right)-\ell_{M}(\zeta)}{2} \\
b_{1} & =\frac{\ell_{M}\left(\xi^{\prime}\right)}{2} \\
b_{2} & =\frac{\ell_{M}(v)+\ell_{M}(\zeta)}{2} \\
b_{3} & =\frac{\ell_{M}(v)-\ell_{M}(\zeta)}{2}
\end{aligned}
$$

Unlike in Corollary A.1, the function $f$ may have more than one positive zero, but the number of zeros is clearly finite, which implies a finite number of possible $\lambda$. This completes the proof.

\section{References}

[1] W Abikoff, The real analytic theory of Teichmüller space, Lecture Notes in Math. 820, Springer, Berlin (1980) MR590044

[2] A Baragar, On the unicity conjecture for Markoff numbers, Canad. Math. Bull. 39 (1996) 3-9 MR1382484

[3] A F Beardon, The geometry of discrete groups, Graduate Texts in Math. 91, Springer, New York (1995) MR1393195 Corrected reprint of the 1983 original 
[4] R Brown, Topology, second edition, Ellis Horwood Series: Math. and its Applications, Ellis Horwood Ltd., Chichester (1988) MR984598

[5] P Buser, Geometry and spectra of compact Riemann surfaces, Progress in Math. 106, Birkhäuser, Boston (1992) MR1183224

[6] P Buser, K-D Semmler, The geometry and spectrum of the one-holed torus, Comment. Math. Helv. 63 (1988) 259-274 MR948781

[7] J O Button, The uniqueness of the prime Markoff numbers, J. London Math. Soc. (2) 58 (1998) 9-17 MR1666058

[8] J O Button, Markoff numbers, principal ideals and continued fraction expansions, J. Number Theory 87 (2001) 77-95 MR1816037

[9] A J Casson, S A Bleiler, Automorphisms of surfaces after Nielsen and Thurston, London Math. Soc. Student Texts 9, Cambridge University Press (1988) MR964685

[10] H Cohn, Approach to Markoff's minimal forms through modular functions, Ann. of Math. (2) 61 (1955) 1-12 MR0067935

[11] H Cohn, Representation of Markoff's binary quadratic forms by geodesics on a perforated torus, Acta Arith. 18 (1971) 125-136 MR0288079

[12] G Frobenius, Uber die Markovschen Zahlen, Preuss. Akad. Wiss. Sitzungsberichte (1913) 458-487

[13] T Gauglhofer, K-D Semmler, Trace coordinates of Teichmüller space of Riemann surfaces of signature $(0,4)$, Conform. Geom. Dyn. 9 (2005) 46-75 MR2133805

[14] W M Goldman, The modular group action on real SL(2)-characters of a one-holed torus, Geom. Topol. 7 (2003) 443-486 MR2026539

[15] D Griffiths, At most 27 length inequalities define Maskit's fundamental domain for the modular group in genus 2, from: "The Epstein birthday schrift", Geom. Topol. Monogr. 1, Geom. Topol. Publ., Coventry (1998) 167-180 MR1668355

[16] A Haas, Diophantine approximation on hyperbolic Riemann surfaces, Acta Math. 156 (1986) 33-82 MR822330

[17] U Hamenstädt, Length functions and parameterizations of Teichmüller space for surfaces with cusps, Ann. Acad. Sci. Fenn. Math. 28 (2003) 75-88 MR1976831

[18] S P Kerckhoff, The Nielsen realization problem, Ann. of Math. (2) 117 (1983) 235-265 MR690845

[19] M L Lang, S P Tan, A simple proof of the Markoff conjecture for prime powers, Geom. Dedicata 129 (2007) 15-22 MR2353978

[20] B Maskit, A picture of moduli space, Invent. Math. 126 (1996) 341-390 MR1411137

[21] G McShane, I Rivin, A norm on homology of surfaces and counting simple geodesics, Internat. Math. Res. Notices (1995) 61-69 MR1317643 
[22] M Mirzakhani, Simple geodesics and Weil-Petersson volumes of moduli spaces of bordered Riemann surfaces, submitted (2003)

[23] B Randol, The length spectrum of a Riemann surface is always of unbounded multiplicity, Proc. Amer. Math. Soc. 78 (1980) 455-456 MR553396

[24] I Rivin, Simple curves on surfaces, Geom. Dedicata 87 (2001) 345-360 MR1866856

[25] PC Sarnak, Diophantine problems and linear groups, from: "Proceedings of the International Congress of Mathematicians, Vol. I, II (Kyoto, 1990)", Math. Soc. Japan, Tokyo (1991) 459-471 MR1159234

[26] T A Schmidt, M Sheingorn, Parametrizing simple closed geodesy on $\Gamma^{3} \backslash \mathcal{H}$, J. Aust. Math. Soc. 74 (2003) 43-60 MR1948257

[27] P Schmutz, Systoles of arithmetic surfaces and the Markoff spectrum, Math. Ann. 305 (1996) 191-203 MR1386112

[28] P Schmutz Schaller, Geometry of Riemann surfaces based on closed geodesics, Bull. Amer. Math. Soc. (N.S.) 35 (1998) 193-214 MR1609636

[29] C Series, The geometry of Markoff numbers, Math. Intelligencer 7 (1985) 20-29 MR795536

[30] W Thurston, Minimal stretch maps between surfaces arXiv:math.GT/9801039

Laboratoire Emile Picard, Université Paul Sabatier

Toulouse, France

Section de Mathématiques, École Polytechnique Fédérale de Lausanne SB-IGAT-CTG, BCH, CH-1015 Lausanne, Switzerland

greg.mcshane@gmail.com, hugo.parlier@epfl.ch

Proposed: Benson Farb

Seconded: Dave Gabai, Jean-Pierre Otal
Received: 25 July 2007

Revised: 1 May 2008 